Mon. Not. R. Astron. Soc. 000,1-?? (...) Printed 21 January $2019 \quad$ (MN LATEX style file v2.2)

\title{
Chemical compositions of giants in the Hyades And Sirius superclusters
}

\author{
P. Ramya, ${ }^{1,2 \star}$ Bacham E. Reddy ${ }^{1}$ and David L. Lambert ${ }^{2}$ \\ ${ }^{1}$ Indian Institute of Astrophysics, Bengaluru, India-560034 \\ ${ }^{2}$ The W.J. McDonald Observatory \&5 Department of Astronomy, University of Texas at Austin, Austin, TX 78712, USA
}

Accepted ..... Received .... ; in original form ....

\begin{abstract}
An abundance analysis for 20 elements from $\mathrm{Na}$ to $\mathrm{Eu}$ is reported for $34 \mathrm{~K}$ giants from the Hyades supercluster and for $22 \mathrm{~K}$ giants from the Sirius supercluster. Observed giants were identified as highly probable members of their respective superclusters by Famaey et al. (2005, A\&A, 430, 165). Three giants each from the Hyades and Praesepe open clusters were similarly observed and analysed. Each supercluster shows a range in metallicity: $-0.20 \leqslant[\mathrm{Fe} / \mathrm{H}] \leqslant+0.25$ for the Hyades supercluster and $-0.22 \leqslant$ $[\mathrm{Fe} / \mathrm{H}] \leqslant+0.15$ for the Sirius supercluster with the metal-rich tail of the metallicity distribution of the Hyades supercluster extending beyond that of the Sirius supercluster and spanning the metallicity of the Hyades and Praesepe cluster giants. Relative elemental abundances $[\mathrm{El} / \mathrm{Fe}]$ across the supercluster giants are representative of the Galactic thin disc as determined from giants in open clusters analysed in a similar way to our approach. Judged by metallicity and age, very few and likely none of the giants in these superclusters originated in an open cluster: the pairings include the Hyades supercluster with the Hyades - Praesepe open clusters and the Sirius supercluster with the U Ma open cluster. Literature on main sequence stars attributed to the two superclusters and the possible relation to the associated open cluster is reviewed. It is suggested that the Hyades supercluster's main sequence population contains few stars from the two associated open clusters. As suggested by some previous investigations, the Sirius supercluster, when tightly defined kinematically, appears to be well populated by stars shed by the U Ma open cluster.
\end{abstract}

Key words: stars: abundances — stars: moving groups - Galaxy: kinematics and dynamics - Galaxy: disc

\section{INTRODUCTION}

Stellar superclusters are overdensities in the $(U, V, W)$ space defined by Galactic motions where $U, V$ and $W$ are the radial, tangential and vertical stellar velocity component. Such an overdensity may be referred to also as a stellar moving group or a stellar stream. Modern identifications of kinematic over-densities in the Galactic disc began with Eggen (1958a,b,c) who suggested members of a moving group came from a dissolving open cluster (see Eggen 1996, and references therein). With evidence accumulating that stars within an open cluster share a common chemical composition and age but stars within a stellar supercluster have a spread in composition and age, the perfect association of every supercluster with a dissolved open cluster is unlikely. Partial contamination from an open cluster remains a possibility. Origins of a supercluster now include the idea that

* E-mail: ramyap09@gmail.com they may be composed of field stars given a common motion through dynamical perturbations arising from the Galaxy's spiral arms and/or the central bar (Antoja et al. 2010; Sellwood 2014). Finally, a supercluster may result, as do many stellar streams in the Galactic halo, from the accretion and disruption of a satellite galaxy but this would seem to be a remote possibility because the superclusters generally share the rotation of the Galactic disc.

In our first paper on superclusters (Ramya et al. 2016), we presented and discussed chemical compositions of giants identified with the Hercules stream by Famaey et al. (2005). Here, we extend our work to giants of two other superclusters - the Hyades and Sirius superclusters. The present pair differ from the Hercules stream in that their $(U, V, W)$ velocities appear at first sight to be associated with open clusters. The Hyades supercluster's possible association is with the Hyades and Praesepe open clusters. The Sirius supercluster is linked with the U Ma open cluster. In contrast, the Hercules stream has not been similarly tied to an open cluster. 
This difference between the present pair of superclusters and the Hercules stream may be reflected in the chemical compositions of stars in the three systems: some Hyades and Sirius supercluster stars may have chemical compositions representative of the associated open cluster but Hercules stream members are anticipated by theory and shown by observation to have a spread in chemical compositions.

With the publication of trigonometrical parallaxes and proper motions from the Hipparcos satellite, the presence of stellar moving groups among local FGK dwarfs was rediscussed by several authors. Notably, Montes et al. (2001) reconsidered five young kinematic groups with ages spanning from just $20 \mathrm{Myr}$ up to about $600 \mathrm{Myr}$. The two oldest of the five kinematic groups are the focus of this paper: the Hyades supercluster at $600 \mathrm{Myr}$ and the Sirius supercluster at $300 \mathrm{Myr}$, also known as the Ursa Major group. The mean $(U, V, W)$ of the Hyades supercluster is similar to the mean motions of the Hyades and Praesepe open clusters. The mean $(U, V, W)$ of the Sirius supercluster is similar to the motion of the Ursa Major open cluster. (Montes et al. also identified three younger kinematic groups which are not considered here: the Castor moving group at $200 \mathrm{Myr}$, the IC 2391 supercluster at 35-55 Myr and the Local Association at 20-150 Myr associated with several open clusters. The Local Association has also been called the Pleiades moving group). Further advances and discussion of moving groups are to be anticipated with the astrometry from the Gaia satellite - see, for example, Kushniruk, Schirmer \& Bensby (2017). Wielen (1971) from the age distribution of open clusters within 1000 pc showed that $50 \%$ evaporated within 200 Myr, $10 \%$ have a lifetime longer than 500 Myr and only 2\% survive for longer than 1000 Myr. Wielen's estimates suggest that significant evaporation may have occurred from the Hyades and U Ma open clusters: is this population now in their respective superclusters?

Our reexamination of the origins of the Hyades and Sirius superclusters is based on GK giants. An open cluster's contributions of dwarfs and giants to the population of a supercluster may differ substantially. For an open cluster the giants come from stars more massive than the cluster's present FGK dwarfs and the lifetime as a GK giant is much shorter than the lifetime of its main sequence progenitor and much shorter than the lifetime of the remaining FGK dwarfs. In addition, the initial mass function increases with decreasing mass and this further reduces the number of GK giants relative to the number of FGK dwarfs, as is evident from the scarcity of giants in open clusters. The corollary is that a search among a supercluster for evaporated stars from a parent open cluster may be more productive using main sequence stars than giant stars. This conclusion is subject to qualification on account of differing degrees of dilution of the two samples by 'field' giant and 'field' main sequence stars, respectively.

In this paper, we determine the chemical compositions for giant stars identified by Famaey et al. (2005) as belonging to the Hyades and the Sirius superclusters, compare their compositions with previously reported results for these clusters and superclusters and attempt to assess the contributions of the associated open clusters to the superclusters.

\section{THE SAMPLES}

Famaey et al. (2005) applied a maximum-likelihood method to kinematic data assembled from the Hipparcos catalogue in order to identify structures in the $(U, V)$ plane. Six structures were isolated. About $80 \%$ of the giants were placed within three structures: young giants with small velocity dispersions accounting for $10 \%$ of the total sample, highvelocity giants from the thick disc and the halo accounting for a further $11 \%$ and a smooth background in the $(U, V)$ space accounting for $60 \%$ of the Hipparcos sample. The remaining $20 \%$ of the sample was divided between three groups with roughly equal populations but distinctive motions: the Hyades-Pleiades supercluster with mean velocities $(U, V, W)=(-30.3,-20.3,-4.8)$, the Sirius moving group with mean velocities $(U, V, W)=(+6.5,+4.0,-5.8)$ and the Hercules stream with $(U, V, W)=(-42.1,-51.6,-8.1)$. (All quoted velocities are heliocentric values in $\mathrm{km} \mathrm{s}^{-1}$.) Our present sample is drawn from those giants having a high probability of belonging to either the Hyades-Pleiades (here, the Hyades) supercluster or the Sirius supercluster (aka, the U Ma group). Our sample of giants from the Hercules stream was discussed previously (Ramya et al. 2016).

In order to relate the chosen giants to the young stellar kinematic groups isolated by Montes et al., Figure 1 shows the velocities of the individual giants selected by us from Famaey et al., the mean velocities of the Hyades supercluster and the Sirius supercluster from Famaey et al. and the mean velocities for the same kinematic groups from Montes et al. The most recent wavelet analysis of supercluster locations in the $(U, V)$ plane from Gaia DR1 parallaxes, Tycho proper motions and $R A V E$ radial velocities (Kushniruk, Schirmer \& Bensby 2017) places the Hyades supercluster at $(-44,-18)$ with an elongation of about $10 \mathrm{~km} \mathrm{~s}^{-1}$ in $U$. Kushniruk et al. note that previously published $(U, V)$ centroids for the supercluster range from their own to $(-30,-15)$ by Antoja et al. (2012) and Bobylev \& Bajkova (2016). The mean velocity is approximately between the values given by Famaey et al. (2005) and Montes et al. (2001) in Figure 1. The Sirius supercluster is spread out in $U$ and $V$ with the leading component at $(0,8)$ according to Kushniruk et al. but the consensus from earlier estimates compiled by these authors place the $(U, V)$ velocities close to the location given by Famaey et al. (2005).

The two panels of Figure 1 show the clear separation of the Sirius supercluster (the Ursa Major group in Montes et al.'s designation) from the Hyades supercluster members. A comparable separation is found between the Sirius supercluster and the other kinematic groups identified by Montes et al.: the Local Association, the IC 2391 supercluster and the Castor moving group. The mean motions of the Local Association associated with the Pleiades (and other open clusters), the IC 2391 and the Castor group are shown in Figure 1. There is a difference of a few $\mathrm{km} \mathrm{s}^{-1}$ between the mean velocity of the Hyades and of the Sirius supercluster as found by Famaey et al. and by Montes et al.

Montes et al. link their Hyades supercluster to the Hyades and Praesepe clusters. It is their Local Association which they tie to the Pleiades (and other) clusters and for which they offer the alternative name the Pleiades moving group. Famaey et al. adopt the label Hyades-Pleiades supercluster implying a sampling of the Hyades and the Pleiades 
(and not the Praesepe) clusters. Not surprisingly, Figure 1 hints that the Famaey et al.'s mean velocities for the HyadesPleiades supercluster fall between Montes et al.'s values for the Hyades supercluster and the Local Association. It would appear from the distribution of our giants in Figure 1 that our Hyades sample is little contaminated by the Local Association (i.e., the Pleiades and other clusters) and the Castor moving group. Confusion with the IC 2391 group may arise when considering compositions.

Famaey et al. (2005) give membership probabilities for a giant to belong to the Hyades and Sirius superclusters. Stars were selected by us if the probability of membership was greater than 70 per cent with additional constraints including the colour cut off $V-I<1.2$ to avoid M stars and that a star be observable from the W.J. McDonald Observatory during the observing run. The final samples of giants with determined chemical composition include 34 from the Hyades supercluster and 22 from the Sirius supercluster. Their locations in the $(U, V)$ and $(W, V)$ planes are shown in Figure 1 with blue squares for the Hyades and green triangles for the Sirius supercluster stars. Galactic velocities $(\mathrm{U}, \mathrm{V}, \mathrm{W})$ computed from Gaia astrometry provide distributions within essentially identical to those in Figure 1 which are provided from HIPPARCOS astrometry, that is stream membership is not materially affected by our (ie., Famaey et al.'s) choice of HIPPARCOS astrometry.

In addition to members of the superclusters, we observed giants $\gamma, \delta$ and $\epsilon$ Tau from the Hyades open cluster and giants HD 73598, 73665 and 73710 from the Praesepe open cluster. These stars are recognized in Figure 1 by orange circles for Hyades and orange diamonds for Praesepe giants. There are no giants recognized as belonging to the $\mathrm{U}$ Ma cluster.

\section{OBSERVATIONS AND ABUNDANCE ANALYSIS}

Stars in Table 1 for the Hyades supercluster and in Table 2 for the Sirius supercluster were observed with the Robert G. Tull coudé spectrograph at the Harlan J. Smith 2.7 meter telescope at the W.J. McDonald Observatory (Tull et al. 1995). Spectra cover the wavelength range 3800-10000 A but longward of $5800 \AA$ coverage is incomplete because the free spectral range of the echelle exceeds the width of the CCD. The spectral resolving power is about 60000 and the signalto-noise ratio of a typical spectrum is 100 or more over much of the spectrum. Wavelength calibration was provided by observation of a Th-Ar hollow cathode lamp. All reductions were carried out with the software package Image Reduction and Analysis Facility (IRAF) 1

Conversion of spectra to elemental abundances followed the procedures described in our paper on the Hercules stream (Ramya et al. 2016). Model stellar atmospheres are taken from the Kurucz (1998) grid $2^{2}$ The line list is an expanded version of that given by Ramya et al. (2016). The

1 IRAF is distributed by the National Optical Astronomy Observatory, which is operated by the Association of Universities for Research in Astronomy (AURA) under cooperative agreement with the National Science Foundation.

2 http://kurucz.harvard.edu/grids.html
2016 list was extended by adding Fe I and Fe II lines and now includes heavy elements based on the line selection adopted by Reddy, Giridhar \& Lambert $(2012,2013)$. The equivalent width of these lines are measured manually from the spectrum and the abundances are calculated from individual lines using the 2014 version of the LTE spectral line analysis code MOOG (Sneden 1973) force-fitting the abundances to match the equivalent widths. Hyperfine corrections are applied for the elements Sc, V, Mn, Co, Ba and Eu using the blends driver of MOOG. Table 3 lists the selected lines including their solar equivalent widths and the corresponding solar abundance. This Table also gives the mean solar elemental abundance, the mean solar abundance recommended by Asplund et al. (2009) and the (small) difference between the latter recommendation and the mean abundance from our selected lines. The five additional elements Y to Eu were added as tracers of neutron capture synthesis which are not included among the lighter elements considered in our previous paper on the Hercules stream. Addition of the five was in large part stimulated by our paper 'Prospecting for chemical tags among open clusters' (Lambert \& Reddy 2016). The Y II $5289.81 \AA$ and Nd II $4989.92 \AA$ lines give systematically lower abundances at lower temperatures and, hence, are not considered in determining mean abundances for giants with effective temperatures cooler than $4700 \mathrm{~K}$.

Model atmosphere parameters - effective temperature $T_{\text {eff }}$, surface gravity $\log g$, microturbulence $\xi_{t}$ and metallicity $[\mathrm{M} / \mathrm{H}]$ - are estimated in the customary fashion from the Fe I and FeII lines. Results are provided in Tables 1 and 2 for the two superclusters. Uncertainties are estimated to be $\Delta T_{\text {eff }}= \pm 50 \mathrm{~K}, \Delta \log g= \pm 0.2$ dex,$\Delta \xi_{t}= \pm 0.2 \mathrm{~km} \mathrm{~s}^{-1}$ and $\Delta[\mathrm{M} / \mathrm{H}]= \pm 0.1$ dex (see Ramya et al. 2016).

Photometry, as described by Ramya et al. (2016), provides a check on the spectroscopic parameters. Effective temperatures are obtained from the $(V-K)$ colour with uncertainties estimated to be $\pm 40 \mathrm{~K}$. The mean difference between the spectroscopic and photometric temperature is $-49 \pm 141 \mathrm{~K}$ and $+56 \pm 48 \mathrm{~K}$ for the Hyades and Sirius superclusters, respectively. Surface gravities were obtained through the web interface for the PARAM code $3^{3}$ using the PARSEC isochrones (Bressan et al. 2012), as described by Ramya et al. (2016). Surface gravities from the PARAM code differ slightly from spectroscopic values: the mean difference is $0.02 \pm 0.09$ dex and $+0.04 \pm 0.08$ dex for the Hyades and Sirius superclusters, respectively.

With the spectroscopic atmospheric parameters, elemental abundances were estimated for all entries in the line list. Differential abundances $[\mathrm{El} / \mathrm{H}]$ are estimated using the solar abundances from Asplund et al. (2009) and Table 3. Tables 4, 5 and 6 for the giants from the Hyades supercluster give $[\mathrm{Fe} / \mathrm{H}],[\mathrm{Fe} \mathrm{II} / \mathrm{H}]$ and then $[\mathrm{El} / \mathrm{H}]$ with the line-to-line spread $\sigma$ and the number of lines used for the abundance determination. Tables 7, 8 and 9 give the same information for the giants of the Sirius supercluster. Ionization equilibrium (i.e., $[\mathrm{El} \mathrm{I} / \mathrm{H}] \equiv[\mathrm{El} \mathrm{II} / \mathrm{H}]$ ) is necessarily satisfied for $\mathrm{Fe}$ but it may be noted that it is also closely satisfied for $\mathrm{Ti}$ where the mean $[\mathrm{TiI} / \mathrm{H}]-[\mathrm{Ti} \mathrm{II} / \mathrm{H}]$ is $+0.04 \pm 0.05$ dex for both superclusters.

Table 10 gives the atmospheric parameters and the 
Ramya, Reddy and Lambert

abundance information for the giants belonging to the Hyades and Praesepe clusters. Our discussion of the Hyades supercluster relies partly on comparison of the compositions of the giants from the Hyades and Praesepe open clusters with those from the supercluster. Systematic abundance errors are expected to cancel almost exactly in this comparison. Inspection of Table 10 shows that the spread in $[\mathrm{Fe} / \mathrm{H}]$ among the trio from each cluster is 0.03 dex which should indicate the range of measurement uncertainty for giants in each supercluster. It is also of interest to consider how results in Table 10 compare with recent results in the literature for the same giants.

Literature on the Hyades cluster's metallicity is vast. We refer to a sample of recent studies. Dutra-Ferreira et al. (2016) investigated the iron abundance of the three giants and a sample of main sequence stars from the Hyades cluster. The authors used classical model atmospheres with two different ways of selecting the atmospheric parameters and two selections of FeI and FeII lines. Our mean Fe abundance for the three giants is $[\mathrm{Fe} / \mathrm{H}]=+0.14$ or $\log (\mathrm{Fe})=$ 7.58 since our line selection provides a solar Fe abundance of 7.44. This Fe abundance lies between the estimates by Dutra-Ferreira et al. for their two line lists and provided by a method similar to ours. Tabernero, Montes \& González Hernández (2012) analysed Hyades open cluster and supercluster members including the giant $\epsilon$ Tau for which they obtained the Fe abundance 7.67, a value only 0.09 dex greater than our value. Carrera \& Pancino (2011) analysed the three Hyades giants to obtain a mean Fe abundance of 7.61, also in good agreement with our result. There is a similar level of consistency for the Praesepe giants. Our mean Fe abundance is 7.62. Yang, Chen \& Zhao (2015) analyze high-resolution spectra and obtain $[\mathrm{Fe} / \mathrm{H}]=+0.16 \pm 0.06$ from four giants from a line list based on the solar spectrum. Assuming a solar Fe abundance of 7.50, their analysis gives the cluster abundance as 7.66. As Yang et al. remark, Carrera \& Pancino obtained this same mean Fe abundance from three giants. (In the literature, the metallicity $[\mathrm{Fe} / \mathrm{H}]$ is generally given a prominent place in text and tables. Depending on how the calibration of the Fe lines is accomplished, it may be necessary to add the inferred solar $\mathrm{Fe}$ abundance to the quoted $[\mathrm{Fe} / \mathrm{H}]$ to obtain the stellar $\mathrm{Fe}$ abundance in order to effect a fair comparison between studies.)

Stellar ages of our giants are estimated using the PARAM code with Bayesian priors for the initial mass function (the lognormal function from Chabrier 2001) and the star formation rate (constant). Spectroscopic effective temperature and $[\mathrm{Fe} / \mathrm{H}]$ were used as input together with the van Leeuwen's (2007) Hipparcos parallax and the reddeningcorrected $V$ magnitude. Ages are given in Tables 1 and 2 .

\section{THE SUPERCLUSTERS' ORIGINS?}

Proposals regarding a supercluster's origins are open to test through comparisons of compositions and ages for supercluster members and stars from the putative related open clusters. If a supercluster is dominated by stars from an open cluster, there will be a clear uniformity for both composition and age between cluster and supercluster. If a supercluster is generated primarily by dynamical perturbations provided to field stars by the Galaxy's spiral arms and central bar, the supercluster's stars will exhibit the spread in composition and age expected of field stars across the Galactocentric distances at which the perturbations are capable of directing stars to the solar neighbourhood. Given the presence of abundance gradients in the Galaxy, superclusters composed of stars from different Galactocentric distances are expected to have a range of metallicities and, if abundance gradients differ for different elements, stars in a supercluster may be expected to have different abundance ratios. Additionally, the $(U, V, W)$ space occupied by a supercluster and its possibly related open clusters may be populated also by stars unrelated to either stellar grouping. Some of these 'field' stars will be then mistakenly assigned to the supercluster. Observational errors in $(U, V, W)$ can serve a similar role. Our pursuit of the superclusters' origins begins with discussions of compositions of giants studied by us from Famaey et al.'s (2005) selections for the Hyades Sirius superclusters.

Identification of Hyades supercluster giants with the Hyades and Prasepe open clusters presents challenges. The number of giants assigned by Famaey et al. (2005) to the supercluster far exceeds the expected number of stars shed by the two open clusters unless their original populations were far in excess of the present populations. Furthermore, in our sample of giants from the Hyades supercluster, just three and possibly four of the giants have an age consistent with that of the Hyades open cluster. Of the quartet, only two have the $[\mathrm{Fe} / \mathrm{H}]$ of the Hyades giants. All other giants are considerably older than the two open clusters and about half have a lower metallicity than the Hyades and Praesepe cluster giants. A similar conclusion covers the selection of giants in the Sirius supercluster with main sequence stars in the U Ma cluster suggesting an age of $300 \mathrm{Myr}$ and a metallicity of $[\mathrm{Fe} / \mathrm{H}] \simeq 0.0$. All of our giants in the Sirius supercluster appear older than 300 Myr. The three youngest stars with ages of $0.7 \pm 0.2$ Gyr have $[\mathrm{Fe} / \mathrm{H}] \simeq+0.12$ and compositions (i.e., $[\mathrm{El} / \mathrm{Fe}]$ ) similar to the rest of the sample. (There are no giants securely identified with the U Ma open cluster.)

Independent of age and chemical composition estimates, our samples of giants cannot be traced back to a chemically homogeneous open cluster because of the large spread in composition for each supercluster. Giants from the Hyades and Sirius superclusters each span a range in $[\mathrm{Fe} / \mathrm{H}]$, as shown by the histograms in Figure 2, with the former showing the larger range. The principal difference between the two histograms is the high $[\mathrm{Fe} / \mathrm{H}]$ tail to the Hyades supercluster's histogram which is absent from the Sirius supercluster's histogram. Curiously, this tail is centered on the $[\mathrm{Fe} / \mathrm{H}]$ of the giants from the Hyades and Praesepe open clusters. Although this tail might suggest the presence of Hyades open cluster stars among the Hyades supercluster giants, the majority of these giants are much older than the open cluster.

In short, very few of the giants in our selection of stars attributed by Famaey et al. to the Hyades and Sirius superclusters and analyzed here can be identified as originating from an associated open cluster. Thus, the giants of these superclusters owe their origin to either perturbations exercised on residents of the Galactic thin disc and/or contamination of the supercluster by unrelated field stars thanks to measurement errors and possibly too generous criteria for $(U, V, W)$. In these circumstances, it is of interest to com- 
Hyades and Sirius streams

pare the compositions of the giants in the two superclusters with compositions of field giants.

Several large studies of field giants are available for consideration, e.g., Luck \& Heiter (2007), Mishenina et al. (2007, 2013) and Luck (2015). Inspection of plots of abundance ratios $[\mathrm{El} / \mathrm{Fe}]$ versus $[\mathrm{Fe} / \mathrm{H}]$ show systematic differences for some elements (El) among these and between other surveys, particularly for heavy elements and others where few absorption lines are available. In order to minimize systematic differences, we adopt abundance ratios provided by an ongoing survey of giants in open clusters (see Reddy, Lambert \& Giridhar 2016 and papers referenced there). This choice should minimize systematic errors because the analyses of the clusters and ours of the superclusters use very similar line lists and analytical techniques. The sample of clusters covers the range of $[\mathrm{Fe} / \mathrm{H}]$ from about -0.2 to 0.0 because the clusters are primarily in the anti-centre direction. The sample is broken into the two subsamples $[\mathrm{Fe} / \mathrm{H}$ $\geqslant-0.10$ with 18 clusters and $[\mathrm{Fe} / \mathrm{H}] \leqslant-0.10$ with 16 clusters. Our samples of supercluster giants are similarly divided into subsamples. Mean [El/Fe] and their standard deviations are given in Table 11 for the two samples of open clusters, three $[\mathrm{Fe} / \mathrm{H}]$ groupings from the Hyades supercluster and two $[\mathrm{Fe} / \mathrm{H}]$ groupings from the Sirius supercluster.

Table 11 may be used to address three questions with a bearing on the origins of the superclusters. First, do the giants of the Hyades and Sirius superclusters have identical compositions across their common range of $[\mathrm{Fe} / \mathrm{H}]$ ? Second, how do the compositions of the superclusters relate to compositions of the chosen sample of open clusters? Third, do the superclusters at the solar metallicity $([\mathrm{Fe} / \mathrm{H}]=0.0)$ have the solar composition?

The answer to the first of the three questions is that the giants of the two superclusters define a single relation for each $[\mathrm{El} / \mathrm{Fe}]$ to within the typical standard deviation of about \pm 0.06 . There is a hint that the $\mathrm{El} / \mathrm{Fe}$ ] for heavy elements $\mathrm{Y}$ to $\mathrm{Nd}$ that $[\mathrm{El} / \mathrm{Fe}]$ for the Sirius supercluster are marginally greater than for the Hyades supercluster but this is not the case for $\mathrm{Eu}$, the quintessential $r$-process element.

Our second question concerns the relation between the two superclusters and the sample of open clusters where the latter are systematically metal-poor relative to the average giant of the superclusters. Inspection of Table 11 suggests the open clusters are slightly underabundant in Zn, overabundant in $\mathrm{Zr}$, and underabundant in $\mathrm{La}$ and $\mathrm{Nd}$ relative to the sample from the two superclusters but the differences are comparable to the standard deviations. Among the sample of open clusters, there is a spread in $s$-process heavy element abundances at a given $[\mathrm{Fe} / \mathrm{H}]$. Lambert \& Reddy (2016) attribute the spread to different degrees of contamination of a cluster's natal cloud by $s$-process products from AGB stars. A similar spread may be present among giants from the Sirius but not the Hyades supercluster.

The third question involves the comparison between the supercluster giants at $[\mathrm{Fe} / \mathrm{H}]=0.0$ and the solar composition. For almost all elements, the mean $[\mathrm{El} / \mathrm{Fe}]$ at $[\mathrm{Fe} / \mathrm{H}]=$ 0.0 from giants in the two superclusters are within $\pm 0.10 \mathrm{dex}$ corresponding to differences expected from the measurement errors and for 10 of the 14 elements the difference is within $\pm 0.05 \mathrm{dex}$ (see Table 11). One obvious exception in Table 11 is $\mathrm{Na}$ but the overabundance of $\mathrm{Na}$ in giants from the supercluster (and the sample of open clusters) is simply the result of the first dredge-up increasing the $\mathrm{Na}$ abundance in the stellar atmosphere (Karakas \& Lattanzio (2014). Other exceptions include Y (marginally), Ba (marginally), La and $\mathrm{Nd}$ at about $0.2 \mathrm{dex}$ and Eu at about 0.15 dex. Comparisons with solar abundances necessarily incorporate possible systematic errors included in analyses of giant stars including non-LTE effects and the neglect of stellar spots and granulation.

The answers to the three questions suggest that the supercluster's giants are drawn from the Galactic disc rather than their associated open clusters with distinctive compositions, i.e., distinguishing sets of chemical tags. This conclusion affirms the interpretations reached above from the spread in $[\mathrm{Fe} / \mathrm{H}]$ and the ages of the supercluster giants. Given that the samples of supercluster giants may be contaminated by field giants unrelated to the supercluster with its particular $(U, V, W)$, the detailed compositions of giant members of the superclusters seem unlikely to be powerful clues to a supercluster's origin. Perhaps, more information may be provided by detailed analyses of main sequence members of the Hyades and the Sirius superclusters.

\section{OTHER ANALYSES OF THE HYADES AND SIRIUS SUPERCLUSTERS}

In the following, we review published results on the compositions of stars in the two superclusters and compare these results with our own as we attempt to determine contamination of the superclusters by evaporation from associated open clusters.

\subsection{The Hyades supercluster}

The composition of stars comprising the Hyades supercluster and their relation to the Hyades open cluster has been discussed previously by De Silva et al. (2011), Pompéia et al. (2011) and Tabernero et al. (2012). Samples of supercluster stars were chosen according to different but similar $(U, V, W)$ criteria. Assessments of the contamination of the Hyades supercluster by stars from the Hyades and Praesepe open clusters hinge on the chemical and age homogeneity of the open clusters.

Chemical homogeneity for the Hyades open cluster was established at the 0.04 dex level for a selection of elements from $\mathrm{Na}$ to $\mathrm{Nd}$ for F-K dwarfs (Paulson et al. 2003; De Silva et al. 2006). Remarkably, Liu et al. (2016) report that the cluster is 'chemically inhomogeneous' at the 0.02 dex level from analyses of 19 elements from $\mathrm{C}$ to Ba. Even the 0.04 dex limit on inhomogeneities is a minor issue relative to the spread in compositions among supercluster members. Comparable investigations of chemical homogeneity across the Praesepe open cluster have not been reported but the assumption of chemical homogeneity is assumed to prevail.

Ages of stars in the supercluster may be checked against the open clusters' age for which estimates in the literature include $0.7 \pm 0.1$ Gyr (Salaris et al. 2004) and $625 \pm 50 \mathrm{Myr}$ (Perryman et al. 1998) for the Hyades and 0.7 \pm 0.1 Gyr (Salaris et al. 2004) and $590 \mathrm{Myr}$ (Fossati et al. 2008) for Praesepe. If evaporation from the clusters is the dominant contributor to a supercluster, supercluster members' ages 
Ramya, Reddy and Lambert

will be sharply peaked at the open cluster's age. If dynamical perturbations are the key origin, stars in a supercluster will show a spread in ages. Famaey, Siebert \& Jorissen (2008) showed that the spread in ages for the Hyades (and Sirius) supercluster main sequence members arise primarily from dynamical perturbations acting on field disc stars. This result is supported by our sample: just seven of the Hyades supercluster giants in Table 1 have ages of 0.6-0.7 Gyr overlapping with the estimates for the open cluster giants. Of this septet, only one and, perhaps, two have the composition of the Hyades and Praesepe clusters.

In an investigation similar to ours, De Silva et al. (2011) conducted an abundance analysis of 20 giants with mean velocities of the Hyades cluster and with an age consistent with that of the cluster. Just four of the 20 stars had the metallicity of the Hyades cluster. De Silva et al. concluded that the quartet "are likely to be former members of the Hyades open cluster" but this is possibly an overestimate because the compositions (i.e., $[\mathrm{El} / \mathrm{Fe}]$ ) of the quartet were not shown to be distinctly different from that of the other (field) giants in their sample.

Pompéia et al. (2011) analysed 21 main sequence stars drawn from the Geneva-Copenhagen Survey (Nordström et al. 2004) with $(U, V, W)$ velocities representative of the Hyades supercluster. Spectra were also obtained and analysed for five certain and four possible main sequence members of the Hyades open cluster. Abundances were obtained for $\mathrm{Li}, \mathrm{Na}, \mathrm{Mg}, \mathrm{Fe}, \mathrm{Zr}, \mathrm{Ba}, \mathrm{La}, \mathrm{Ce}, \mathrm{Nd}$ and $\mathrm{Eu}$. The authors' adoption of $\mathrm{Li}$ as 'an efficient population tagger' was based on the observation that the lithium abundance along the Hyades main sequence is a well-defined function of effective temperature and, importantly, the Hyades Li relation evolves with age with $\mathrm{Li}$ depletion being more rapid the lower the mass of the main sequence star. The Li relation for the Hyades (which is identical to that of the Praesepe) is taken from Cummings et al. (2017) analysis of a large sample of cluster members. Pompéia et al.'s Li abundances for the five certain and four possible members of the Hyades open cluster fall on the Cummings et al.'s relation (Figure 3). Many of the 21 stars from the supercluster have a clear Li abundance deficiency with respect to an open cluster star of the same effective temperature (Figure 3). Pompéia et al. from a broader consideration of the elemental abundances considered two of the 21 stars to be 'evaporated candidates' and indeed this pair fall on the open cluster's Li relation. Four other supercluster candidates have a Li abundance compatible with the Li relation of the Hyades open cluster but were not tagged as evaporated candidates by the broader consideration of the abundances.

These broader considerations indeed show that the two evaporated candidates among the supercluster members share the abundances of the Hyades cluster members. Especially interesting in this regard is the range in heavy element abundances among Pompéa et al.'s main sequence stars. There is a clear tendency, for example, for $[\mathrm{La} / \mathrm{Fe}]$ to decline with increasing $[\mathrm{Fe} / \mathrm{H}]$. This pattern is repeated for other heavy elements including for $\mathrm{Eu}$, the quintessential $r$ process element. Such a trend is now well established from many studies of main sequence stars in the solar neighbourhood (see, e.g., Battistini \& Bensby (2016)). In Figure 4, we show Pompéia et al.'s differential $[\mathrm{La} / \mathrm{Fe}]$ and $[\mathrm{Nd} / \mathrm{Fe}]$ abundances: these are the abundance ratios with respect to the Hyades cluster member HD 26756. There is a clear correlation between $\Delta[\mathrm{La} / \mathrm{Fe}]$ and $\Delta[\mathrm{Nd} / \mathrm{Fe}]$. This correlation appears largely restricted to heavy elements; $[\mathrm{Mg} / \mathrm{Fe}]$ may be very weakly correlated with $[\mathrm{La} / \mathrm{Fe}]$ (Figure 5 top panel) and $[\mathrm{Zr} / \mathrm{Fe}]$ (Figure 5 bottom panel) is less strongly correlated with $[\mathrm{La} / \mathrm{Fe}]$ than is $[\mathrm{Nd} / \mathrm{Fe}]$. These figures confirm that the two stars identified by Pompéia et al. as evaporated from the Hyades open cluster indeed have the composition of cluster stars. Most of the four stars identified as 'possible' open cluster members do not have the same composition including $[\mathrm{Fe} / \mathrm{H}]$ and heavy element abundances as the open cluster members. Our conclusion echoing that by Pompéia et al. is that the cluster contributed no more than two stars (the evaporated candidates) to this sample of Hyades supercluster main sequence stars.

A different selection of Hyades supercluster members was made by Tabernero et al. (2012) who analysed 62 FGK stars with $(U, V, W)$ within $10 \mathrm{~km} \mathrm{~s}^{-1}$ of the mean velocities of the Hyades supercluster (Montes et al. 2001). The sample was a mix of main sequence and giant stars and included three main sequence stars and the giant $\epsilon$ Tau from the Hyades cluster. Abundances were reported for $\mathrm{Na}, \mathrm{Mg}, \mathrm{Al}$, $\mathrm{Si}, \mathrm{Ca}, \mathrm{Sc}, \mathrm{Ti}, \mathrm{V}, \mathrm{Cr}, \mathrm{Cu}, \mathrm{Zn}, \mathrm{Y}, \mathrm{Zr}, \mathrm{Ba}, \mathrm{Ce}$ and Nd. The Hyades cluster's Fe abundance was reported as $[\mathrm{Fe} / \mathrm{H}]=$ +0.21 from $\epsilon$ Tau and +0.10 from the three dwarf members.

Supercluster stars were identified as evaporated stars from the Hyades cluster, firstly, if their $[\mathrm{Fe} / \mathrm{H}]$ fell within the interval $-0.05 \leqslant[\mathrm{Fe} / \mathrm{H}] \leqslant+0.16$ and, secondly, if the $[\mathrm{El} / \mathrm{Fe}]$ of the various elements also matched the values of Hyades cluster members. Tabernero et al. concluded primarily from the $[\mathrm{Fe} / \mathrm{H}]$ determinations that 28 of their sample of 62 (i.e., $46 \%$ ) supercluster stars had been shed by the Hyades open cluster. This is likely an overestimate because the 0.20 dex width of the $[\mathrm{Fe} / \mathrm{H}]$ window seems generous in light of the homogeneity of Hyades cluster and the precision of the abundance analysis.

In light of the heavy element abundances for Pompéia et al.'s sample (Figure 5), we present Figure 6 where $[\mathrm{Ce} / \mathrm{Fe}]$ and $[\mathrm{Nd} / \mathrm{Fe}]$ are compared for Tabernero et al' $\mathrm{s}$ sample of Hyades cluster and supercluster stars. (Giants are offset from the sequence defined by main sequence stars indicating systematic differences in abundances between dwarfs and giants.) For main sequence supercluster stars, there appears to be a continuous run from low $[\mathrm{Ce} / \mathrm{Fe}]$ and $[\mathrm{Nd} / \mathrm{Fe}]$ to high $[\mathrm{Ce} / \mathrm{Fe}]$ and $[\mathrm{Nd} / \mathrm{Fe}]$ which is similar to but weaker than the trend in Figure 5. The three Hyades open cluster dwarfs fall on the relation defined by the other stars. A concentration of the supercluster stars with $[\mathrm{Fe} / \mathrm{H}]$ within the adopted $[\mathrm{Fe} / \mathrm{H}]$ window surround the trio of Hyades open cluster main sequence members but some of these stars may not have the same abundances as cluster members. This concentration approximates the $46 \%$ considered by Tabernero et al. to have belonged to the Hyades open cluster. However, it has not been shown either that these stars have distinctive chemical tags enabling evaporated open cluster stars to be distinguished from field stars or that their ages match the age of the Hyades open cluster. In fact, the mean [El/Fe] for stars with $[\mathrm{Fe} / \mathrm{H}]$ within the designated window for cluster membership and for the three open cluster dwarfs is within a standard deviation of \pm 0.10 dex of solar values (i.e., 0.0 ) for all elements but for $\mathrm{V}$ which is noted as a problematic element by Tabernero et al. A comparable remark applies to 
Hyades and Sirius streams

the comparison between our $[\mathrm{El} / \mathrm{Fe}]$ for the Hyades supercluster giants and Tabernero et al.'s results for their main sequence stars.

In seeking to apply the lithium abundance test employed by Pompéia et al., we searched for Li abundance determinations for the main sequence stars in Tabernero et al.'s sample. (Thanks to a giant's deep convective envelope causing severe $\mathrm{Li}$ dilution, $\mathrm{Li}$ is not an effective chemical tag among giants.) Through SIMBAD, we searched for determinations of the $\mathrm{Li}$ abundance. Determinations or upper limits were found for 45 stars of which 25 satisfied and 20 failed to meet Tabernero et al.'s $[\mathrm{Fe} / \mathrm{H}]$ constraint to be considered to be an evaporated cluster member. Figure 7 shows the $\mathrm{Li}$ abundances as a function of effective temperature. With a single exception of V686 Per (Xing \& Xing 2012) with an abnormally high $\mathrm{Li}$ abundance, the smooth run of $\mathrm{Li}$ abundance with effective temperature includes essentially all stars irrespective of their $[\mathrm{Fe} / \mathrm{H}]$ (i.e., members with $[\mathrm{Fe} / \mathrm{H}]$ within the chosen window and nonmembers with $[\mathrm{Fe} / \mathrm{H}]$ below and above the $[\mathrm{Fe} / \mathrm{H}]$ - compare Figure 3 and Figure $7)$. This result may confirm that all the main sequence stars have a similar age. However, lithium depletion is not particularly rapid. Bubar \& King (2010) present Li-T eff $_{\text {plots }}$ for the Pleiades, Hyades, NGC 752 and M 67. At $6000 \mathrm{~K}$, for example, the Li abundance in the Hyades main sequence members is 2.8 at the age of $625 \mathrm{Myr}$ and has dropped to only 2.6 in NGC 752 at the age of 2.5 Gyr and to 2.4 in M 67 at the age of 5 Gyr. Thus, lithium is not an especially precise chronometer.

In summary, Tabernero et al.'s selection of Hyades supercluster main sequence candidates may include stars evaporated from the Hyades and Praesepe open clusters. But the adopted $[\mathrm{Fe} / \mathrm{H}]$ window seems too relaxed to ensure that only stars from the chemically homogeneous Hyades (and Praesepe) clusters are securely identified in the absence of chemical tags distinguishing cluster from field stars. The $\mathrm{Li}$ abundances suffice to eliminate a couple of stars within the adopted $[\mathrm{Fe} / \mathrm{H}]$ range as older than the Hyades cluster. Tighter $(U, V, W)$ criteria should be helpful in identifying evaporated stars within the supercluster.

\subsection{The Sirius supercluster and the U Ma cluster}

The Sirius supercluster with kinematic brethren across the sky is associated with "a compact nucleus, similar in size to an ordinary loose galactic cluster" (Roman 1949) in the U Ma constellation. Main sequence stars have been identified with the nucleus (i.e., the U Ma open cluster) - see Roman (1949, Tables 11 and 14), Soderblom \& Mayor (1993, Table 6 ) and King et al. (2003, Table 5). Recent abundance analyses of stars in the U Ma nucleus show a near-solar metallicity, e.g., Monier (2005) from three F stars obtained a mean $[\mathrm{Fe} / \mathrm{H}]$ of -0.10 , Ammler-von Eiff \& Guenther (2009) from four $\mathrm{F}$ stars obtained a mean $[\mathrm{Fe} / \mathrm{H}]$ of -0.04 and Tabernero et al. (2017) from three $\mathrm{F}$ stars got a mean $[\mathrm{Fe} / \mathrm{H}]$ of -0.07 .

As an extension of these concordant abundance analyses, it is of interest to explore the possible relationship between the open cluster and the Sirius supercluster. King \& Schuler (2005) compiled results from the literature and analysed several additional stars to provide spectroscopic $[\mathrm{Fe} / \mathrm{H}]$ for 17 main sequence stars. This sample with membership probabilities from King et al. (2003) included two from the
U Ma nucleus and fifteen attributed to the supercluster including stars very far from the $\mathrm{U}$ Ma nucleus. The mean $[\mathrm{Fe} / \mathrm{H}]$ for the 17 is $[\mathrm{Fe} / \mathrm{H}]=-0.06 \pm 0.07$. In the $(U, V)$ plane, the sample is centered within a few $\mathrm{km} \mathrm{s}^{-1}$ of the mean $(U, V)$ of the nucleus at $(+13.9 \pm 0.6,+2.9 \pm 0.9)$ with just a couple of outliers. The $\mathrm{Li}$ abundances as a function of effective temperature followed the expected relation for a population of stars having the age of the U Ma nucleus. In addition, the selected member stars fell on the predicted isochrone in a colour-magnitude diagram. Since these supercluster members and the $\mathrm{U}$ Ma cluster have the same metallicity, an impression is given that the supercluster and open cluster are intimately related.

Ammler-von Eiff \& Guenther (2009) compiled a list of supercluster members from primarily kinematic assessments, particularly those by Montes et al. (2001) and King et al. (2003). Iron and magnesium abundances were obtained from high-resolution spectra for 17 solar-like stars, four in the nucleus and thirteen in the supercluster. Selected stars were tightly clustered around the $(U, V)$ velocities of the nucleus, say within $\pm 2 \mathrm{~km} \mathrm{~s}^{-1}$ of the centroid $(+13,+3)$. Four stars in the U Ma nucleus gave $[\mathrm{Fe} / \mathrm{H}]=-0.04 \pm 0.08$ and $[\mathrm{Mg} / \mathrm{Fe}]=-0.02 \pm 0.05$. Thirteen stars in the Sirius supercluster gave $[\mathrm{Fe} / \mathrm{H}]=-0.04 \pm 0.04$ and $[\mathrm{Mg} / \mathrm{Fe}]$ $=+0.01 \pm 0.03$. Thus, this study indicates that the $\mathrm{U}$ Ma nucleus and the supercluster, as so tightly defined by $(U, V)$, have identical abundances of $\mathrm{Mg}$ and Fe to within the tight measurement uncertainties. Also, the Li abundances are as anticipated for a population of young coeval main sequence stars. Thus, these stars with common Galactic velocities but with positions spread across the sky may have been shed by the U Ma nucleus.

Tabernero et al. (2017) chose main sequence stars belonging to the Sirius supercluster using more relaxed kinematical criteria than used by King \& Schuler and by Ammler-von Eiff \& Guenther, namely, a star was accepted as a member if its $(U, V, W)$ were within $10 \mathrm{~km} \mathrm{~s}^{-1}$ of the mean velocities of the $\mathrm{U}$ Ma nucleus as determined by King et al. (2003). High-resolution spectra provided abundances for 20 elements for 45 main sequence stars including three from the U Ma nucleus. Tabernero et al.'s sample includes eight stars analysed by Ammler-von Eiff \& Guenther. The two analyses yield consistent results for $[\mathrm{Fe} / \mathrm{H}]$ and $[\mathrm{Mg} / \mathrm{Fe}]$ : mean $[\mathrm{Fe} / \mathrm{H}]$ are $-0.03 \pm 0.06$ and $-0.04 \pm 0.05$ from Ammler-von Eiff \& Guenther and Tabernero et al., respectively. Similar agreement occurs for $[\mathrm{Mg} / \mathrm{Fe}]$.

For Tabernero et al.'s sample with its relaxed criteria for $(U, V, W)$, metallicities $[\mathrm{Fe} / \mathrm{H}]$ were not solely concentrated on the $[\mathrm{Fe} / \mathrm{H}]$ of the $\mathrm{U}$ Ma cluster but ranged from -0.37 to +0.23 (see their Figure 2), i.e., a much broader range than found by King \& Schuler and Ammler-von Eiff \& Guenther. Inspection shows that $[\mathrm{Fe} / \mathrm{H}]$ declines with decreasing effective temperature: 26 stars hotter than $5500 \mathrm{~K}$ give $[\mathrm{Fe} / \mathrm{H}]$ $=+0.05 \pm 0.05$ and eight stars cooler than $5500 \mathrm{~K}$ give $[\mathrm{Fe} / \mathrm{H}]=-0.06 \pm 0.04$. By inspection of their Figure 6 , it appears that most elements show a rather similar decrease in $[\mathrm{El} / \mathrm{H}]$ with temperature and, thus, this systematic effect may have little influence on the ratios $[\mathrm{El} / \mathrm{Fe}]$. The three stars from the U Ma nucleus (open cluster) and the supercluster stars with the $[\mathrm{Fe} / \mathrm{H}]$ of the $\mathrm{U}$ Ma nucleus have very similar [El/Fe]: differences between the supercluster and the nucleus are within \pm 0.07 dex for the great majority of the 
Ramya, Reddy and Lambert

elements. Tabernero et al. considered stars with $[\mathrm{Fe} / \mathrm{H}]$ between -0.10 and +0.12 to be evaporated members of the $\mathrm{U}$ Ma open cluster. Stars with $[\mathrm{Fe} / \mathrm{H}]$ outside these limits were considered not to have come from the cluster. The 34 stars inside the $[\mathrm{Fe} / \mathrm{H}]$ bounds gave a mean $[\mathrm{Fe} / \mathrm{H}]=0.03 \pm 0.06$. Members and nonmembers are not clearly distinguishable by their $(U, V, W)$.

For an application of the lithium test, lithium abundances were retrieved from SIMBAD for 32 of the sample of 45 main sequence stars. Of the 32 , all but nine had their $[\mathrm{Fe} / \mathrm{H}]$ within the range adopted by Tabernero et al. for membership of the $\mathrm{U}$ Ma cluster. The stars in the $\mathrm{U} \mathrm{Ma}$ cluster's nucleus are included in the sample. In addition, additional supercluster members with a $\mathrm{Li}$ abundance determination were taken from Ammler-von Eiff \& Guenther. The Li- $\mathrm{T}_{\text {eff }}$ relation is shown in Figure 8 together with the Li abundances for Hyades oepn cluster stars from Cummings et al. (2017). Maximum Li abundances for the Sirius supercluster stars run on the high $\mathrm{Li}$ abundance side of the Hyades relation below about $T_{\text {eff }} \leqslant 5500 \mathrm{~K}$; the U Ma cluster is younger than the Hyades and Praesepe open clusters. A few stars with $[\mathrm{Fe} / \mathrm{H}]$ consistent with $\mathrm{U}$ Ma cluster membership have a $\mathrm{Li}$ abundance below the $\mathrm{Li}-\mathrm{Teff}$ relation for the Sirius supercluster. These Li-poor supercluster stars are unlikely to have come from the U Ma cluster. Other stars have a Li abundance consistent with the idea that they originated in the U Ma cluster.

In summary, compositions of the main sequence stars selected by King \& Schuler (2005) and by Ammler-von Riff \& Guenther (2009) with their tight kinematical criteria suggest that the Sirius supercluster with members widely distributed across the sky suggest that many were evaporated from the U Ma open cluster. A similar less certain conclusion seems supportable from the sample of main sequence stars chosen and analysed by Tabernero et al. with less restrictive requirements on the $(U, V, W)$ for supercluster membership.

\section{CONCLUDING REMARKS}

The investigation in this paper concerns the origins of stars in the Hyades and the Sirius superclusters and, more particularly, to what degree these superclusters are populated by stars evaporated from the open clusters commonly associated with them, that is the Hyades and Praesepe clusters with the Hyades supercluster and the U Ma cluster with the Sirius supercluster? In the attempt to answer the question, we have determined the chemical compositions of 34 giants in the Hyades supercluster and 22 giants in the Sirius supercluster and rediscussed published abundance analyses of main sequence and giant stars of the Hyades supercluster and the putative associated Hyades and the Praesepe open clusters and main sequence stars of the Sirius supercluster and its associated U Ma open cluster.

In principle, the chemical compositions of supercluster members may test the idea that stars evaporated from associated open clusters reside in a supercluster. If the idea is strictly valid, stars in the open cluster and supercluster will be coeval and the composition of stars belonging to the cluster and supercluster will be identical. (Given that systematic errors may afflict abundance analyses, the test is most securely applied to stars of the same type in the cluster and the supercluster analysed in the same way.) The test relies on two assumptions: (i) stars presently in and those evaporated from the parent cluster have identical compositions and ages and (ii) the open cluster has distinctive chemical tags enabling its stars to be distinguished from other potential contributors or contaminants to the supercluster. Relevant to (i) are observations that open clusters are chemically homogeneous to about 0.04 dex for all elements other than Li. In the case of main sequence stars, lithium may serve as a chemical tag, as was applied by Pompéia et al. (2011) to a sample of Hyades supercluster candidates (see their Figure 11). Of course, this lithium test does not apply to giant stars. (The statement about chemical homogeneity of a cluster requires obvious qualifiers excluding such stars as the peculiar A and F main sequence stars.) With respect to (ii), possible contributors are field stars from elsewhere in the Galactic disc. Uncertainties in stellar distances, radial velocities and proper motions disperse stars in the $(U, V, W)$ space and may result in accidental mixing of stars into the supercluster from the associated open cluster and the population of field stars. In a sample of giants attributed to a supercluster, the fraction of stars evaporated from the associated open cluster is more likely to be diluted by field stars than a sample of main sequence stars.

Judged by chemical composition and age, our sample of giants from the Hyades supercluster contains very few and perhaps no stars from the Hyades and Praesepe open clusters. Notably, the compositions of six giants from the two open clusters are at the upper end of the metallicity spread of the Hyades supercluster. The $[\mathrm{Fe} / \mathrm{H}]$ spread among the supercluster's stars is real and not a reflection of measurement errors; compare the spread with the uniformity of the $[\mathrm{Fe} / \mathrm{H}]$ of the Hyades and of the Praesepe giants. Giants from the Sirius supercluster span a smaller range in $[\mathrm{Fe} / \mathrm{H}]$ than their counterparts in the Hyades supercluster but, nonetheless, the spread in composition and age are incompatible with the proposal that our sample of Sirius supercluster giants is fed to a large degree by evaporation from the U Ma cluster.

In contrast, analyses of main sequence members of the superclusters may suggest the presence of stars evaporated from the associated open clusters. The most convincing evidence is provided for the Sirius supercluster and the associated $\mathrm{U}$ Ma open cluster. When the selection by $(U, V, W)$ is as tight as insisted upon by Ammler-von Eiff \& Guenther (2009) and King \& Schuler (2005) for the Sirius supercluster, population of the supercluster by evaporation from the U Ma open cluster becomes a very likely prospect, as these authors recognized. It has yet to be shown that the Hyades supercluster contains appreciable numbers of stars from the Hyades and Praesepe clusters. Pompéia et al. (2011) suggested pollution of the Hyades supercluster from these open clusters was minimal. Studies by Tabernero et al. (2012, 2017) of main sequence stars attributable to the Hyades and Sirius superclusters suggest that significant fractions of the stars in the superclusters were provided by host open clusters. Tabernero et al. (2012) in their abstract remark that " $46 \%$ of our candidates are members" of the Hyades open cluster. For the Sirius supercluster and the U Ma open cluster, Tabernero et al. (2017) note that 29 out of 44 (i.e., $66 \%$ ) of their supercluster members are "likely to originate from a dispersing cluster" (i.e., U Ma). These estimates of open cluster contamination of the two superclusters depend on the 
width of the $[\mathrm{Fe} / \mathrm{H}](\simeq 0.2 \mathrm{dex})$ windows taken as consistent with the $[\mathrm{Fe} / \mathrm{H}]$ of the open cluster which seem generous assessments of measurement errors and far exceed the small dispersion in $[\mathrm{Fe} / \mathrm{H}]$ within an open cluster. These windows are also contaminated as a result of the more relaxed the $(U, V, W)$ windows.

To refine further our understanding of the origins of the Hyades and Sirius superclusters will call for tighter definitions of the $(U, V, W)$ velocities of these superclusters and their fine structure - see Kushniruk et al.'s (2018) discussion based on Gaia's astrometric data and RAVE's radial velocities (and other measurements). Determination of chemical compositions with a focus on high precision should pay particular attention to the heavy elements (say Y to Eu) and to Li. With trigonometrical parallaxes from Gaia and stellar evolutionary tracks it may be possible to invoke age as an important parallel indicator for many more main sequence stars than at present. Refinement of a supercluster's stars with regards to membership, kinematics, detailed composition and ages should aid in determining the supercluster's origin and whether certain open clusters have contributed to the supercluster.

\section{ACKNOWLEDGMENTS}

DLL thanks the Robert A. Welch Foundation of Houston, Texas for support through grant F-634. We thank the referee for a constructive report.

\section{REFERENCES}

Ammler-von Eiff M., Guenther E. W., 2009, A\&A, 508, 677 Antoja T., Figueras F., Torra J., et al. 2010, Lecture Notes and Essays in Astrophysics, 4, 13

Antoja T., Helmi A., Bienayme O., et al. 2012, MNRAS, 426, L1

Asplund M., Grevesse N., Sauval A. J., et al. 2009, ARA\&A, 47, 481

Battistini C., Bensby T., 2016, A\&A, 586, A49

Bobylev V. V., Bajkova A. T., 2016, Astronomy Letters, 42, 90

Bressan A., Marigo P., Girardi L., et al. \& Nanni A., 2012, MNRAS, 427, 127

Bubar E. J., King J. R., 2010, AJ, 140, 293

Carrera R., Pancino E., 2011, A\&A, 535, A30

Chabrier G., 2001, ApJ, 554, 1274

Cummings J. D., Deliyannis C. P., Maderak R. M., et al. 2017, AJ, 153, 128

De Silva G. M., Sneden C., Paulson D. B., et al. 2006, AJ, 131, 455

de Silva G. M., Freeman K. C., Bland-Hawthorn J., et al. 2011, MNRAS, 415, 563

Dutra-Ferreira L., Pasquini L., Smiljanic R., et al. 2016, A\&A, 585, A75

EggenO. J.. 1958a, Observatory, 78, 21

Eggen O. J., 1958b, MNRAS, 118, 65

Eggen O. J., 1958c, MNRAS, 118, 154

Eggen O. J., 1996, AJ, 112, 1595

Famaey B., Jorissen A., Luri X., Mayor M., Udry S., Dejonghe H., Turon C., 2005, A\&A, 430, 165
Famaey B., Siebert A., Jorissen A., 2008, A\&A, 483, 453

Fossati L., Bagnulo S., Landstreet J., et al. 2008, A\&A, 483, 891

Karakas A. I., Lattanzio J. C., 2014, mboxPubl. Astron. Soc. Australia, 31, e030

King J. R., Villarreal A. R., Soderblom D. R., et al. 2003, AJ, 125, 1980

King J. R., Schuler S. C., 2005, mboxPASP, 117, 911

Kurucz R.L., 1998, http://kurucz.harvard.edu/ (online data)

Kushniruk I., Schirmer T., Bensby T., 2017, A\&A, 608, A73

Kushniruk I., Schirmer T., Bensby T., 2018, in Chiappini C., Minchev I., Starkenburg E., Valentini M., eds, Rediscovering Our Galaxy Vol. 334 of IAU Symposium, Kinematic structures found with Gaia DR1/TGAS and RAVE data in the Solar neighbourhood. pp 323-324

Lambert D. L., Reddy A. B. S., 2016, ApJ, 831, 202

Liu F., Yong D., Asplund M., Ramírez I., Meléndez J., 2016, MNRAS, 457, 3934

Luck R. E., Heiter U., 2007, AJ, 133, 2464

Luck R. E., 2015, AJ, 150, 88

Mishenina T. V., Gorbaneva T. I., Bienaymé O., et al. 2007, Astronomy Reports, 51, 382

Mishenina T. V., Pignatari, M., Korotin, S. A., et al., 2013, A\&A, 552, A128

Monier R., 2005, A\&A, 442, 563

Montes D., López-Santiago J., Gálvez M. C., et al. 2001, MNRAS, 328, 45

Nordström B., Mayor M., Andersen J., et al. 2004, A\&A, 418, 989

Paulson D. B., Sneden C., Cochran W. D., 2003, AJ, 125, 3185

Perryman M. A. C., Brown A. G. A., Lebreton Y., et al. 1998, A\&A, 331, 81

Pompéia L., Masseron T., Famaey B., et al. 2011, MNRAS, 415,1138

Ramya P., Reddy B. E., Lambert D. L., Musthafa M. M., 2016, MNRAS, 460, 1356

Reddy A. B. S., Giridhar S., Lambert D. L., 2012, MNRAS, 419, 1350

Reddy A. B. S., Giridhar S., Lambert D. L., 2013, MNRAS, 431, 3338

Reddy A. B. S., Lambert D. L., Giridhar S., 2016, MNRAS, 463, 4366

Roman N. G., 1949, ApJ, 110, 205

Salaris M., Weiss A., Percival S. M., 2004, A\&A, 414, 163

Sellwood J. A., 2014, Rev. Mod. Phys., 86, 1

Sneden C., 1973, PhD Thesis, University of Texas, Austin Soderblom D. R., Mayor M., 1993, AJ, 105, 226

Tabernero H. M., Montes D., González Hernández J. I., 2012, A\&A, 547, A13

Tabernero H. M., Montes D., González Hernández J. I., et al. 2017, A\&A, 597, A33

Tull R. G., MacQueen P. J., Sneden C., Lambert D. L., 1995, PASP, 107, 251

van Leeuwen F., 2007, A\&A, 474, 653

Wielen R., 1971, A\&A, 13, 309

Yang X. L., Chen Y. Q., Zhao G., 2015, AJ, 150, 158

Xing L.-F., Xing Q.-F., 2012, A\&A, 537, A91 

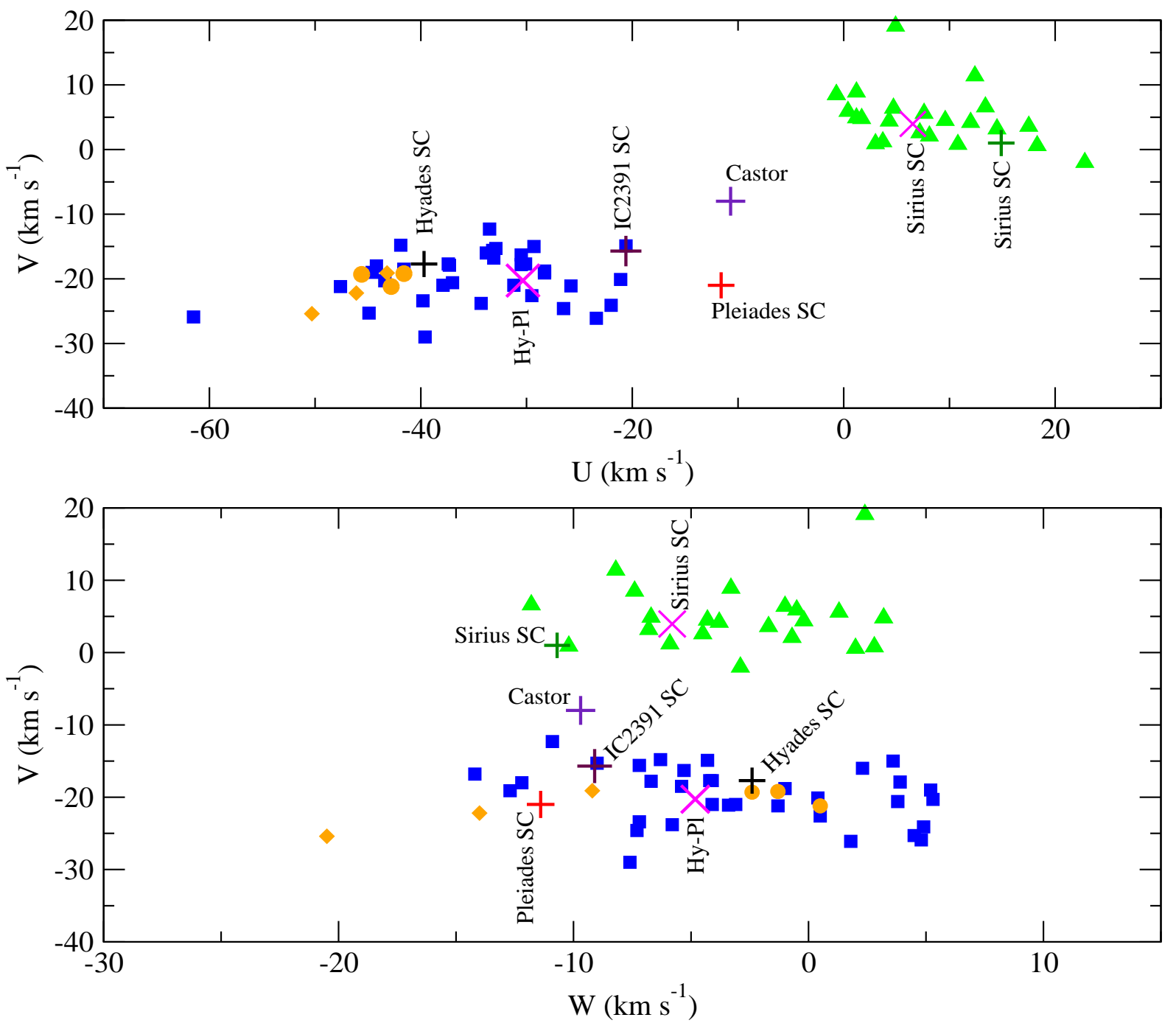

Figure 1. The $(U, V)$ and $(W, V)$ plots. Blue squares and green triangles represent the observed giants from the Hyades and Sirius superclusters, respectively. Orange circles and orange diamonds represent the Hyades open cluster giants and Praesepe open cluster giants, respectively. The + symbols represent the position of five superclusters or moving groups given in Montes et al. (2001): Sirius, Castor, Hyades, IC 2391 and the Local Association. The Magenta crosses represent the position of the Hyades and Sirius superclusters as given in Famaey et al. (2005). 

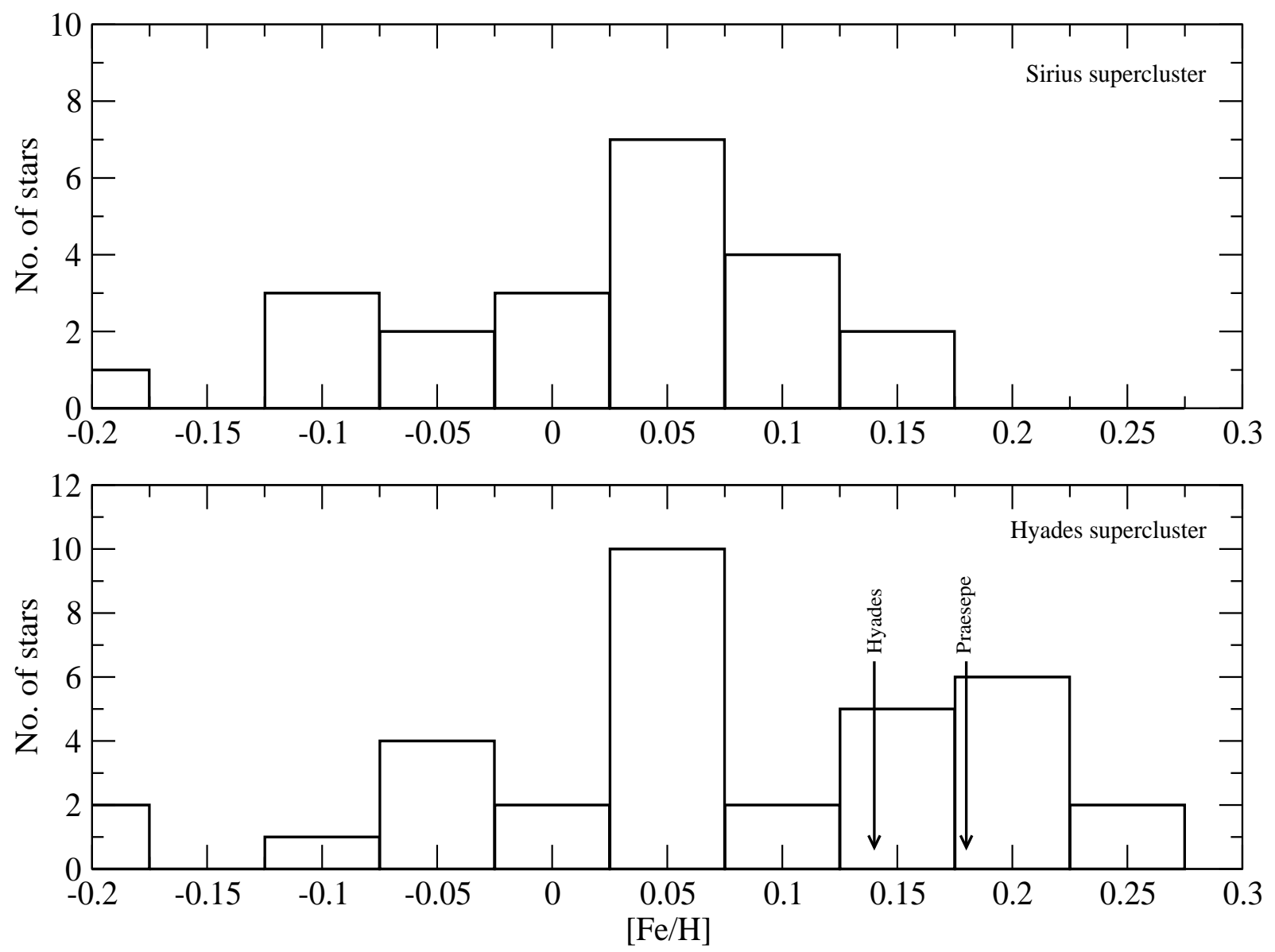

Figure 2. Histograms for $[\mathrm{Fe} / \mathrm{H}]$ for the sample of the 34 giants from the Hyades supercluster (bottom panel) and the 22 giants from the Sirius supercluster (top panel). Mean abundances of the giants from the Hyades and Praesepe open clusters are marked by downward pointing arrows in the bottom panel. 


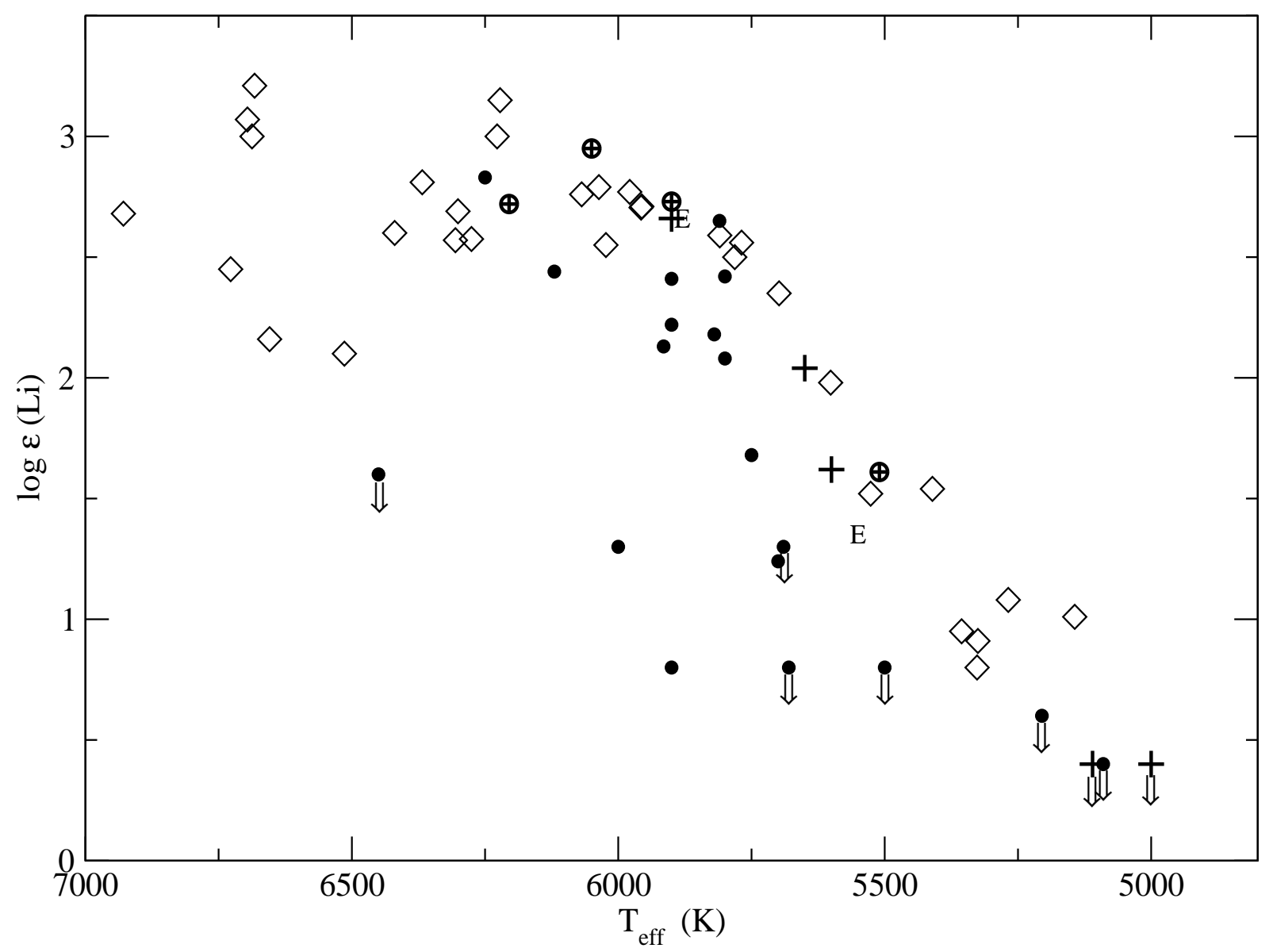

Figure 3. The Lithium abundance $\log \epsilon(\mathrm{Li})$ and effective temperature $\mathrm{T}_{\text {eff }}$ for Hyades supercluster main sequence candidates selected and analysed by Pompéia et al. (2011). Lithium abundances for members of the Hyades (diamonds) open cluster are taken from Cummings et al. (2017). Stars designated by Pompéia et al. as 'certain members' of the Hyades open cluster are represented by the + symbol, their possible members of the open cluster are represented by a encircled + , and two possible evaporated cluster stars are shown by the letter E. Supercluster stars which by their (lower) Li abundance have not been provided by the open clusters are shown as filled circles. The down arrows mark upper limits on the Li abundance 


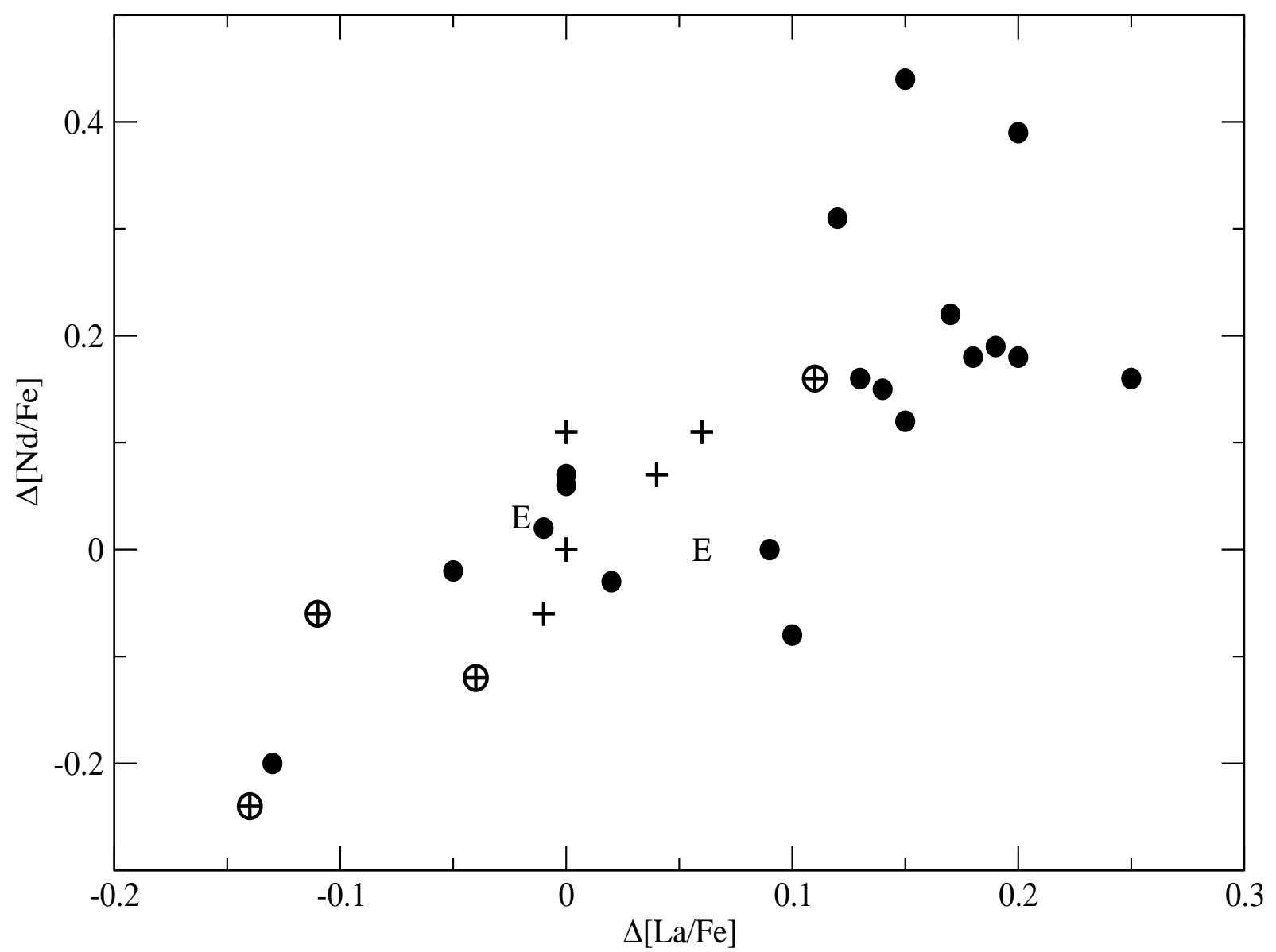

Figure 4. The run of the differential abundance $\Delta[\mathrm{Nd} / \mathrm{Fe}[$ with $\Delta[\mathrm{La} / \mathrm{Fe}]$ for the stars in the Hyades open cluster and supercluster analysed by Pompéia et al. (2011). Abundance ratios are referenced to the values obtained for the Hyades cluster member HD 26756. The symbols distinguish certain $(+$ symbol) and possible $($ encircled +$)$ Hyades open cluster members, possible evaporated cluster stars (capital E) among the supercluster's members and other members of the supercluster (filled circles). 

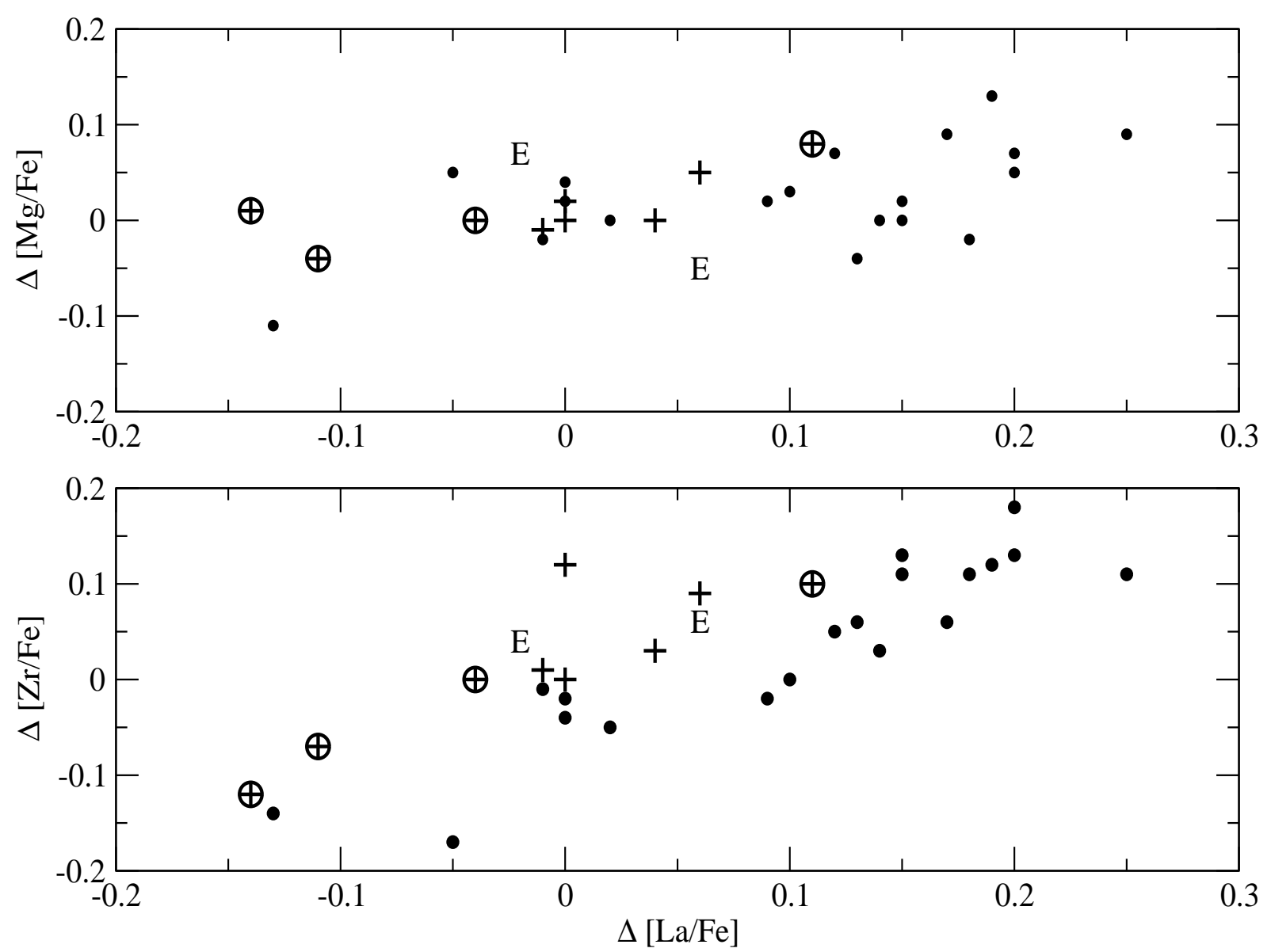

Figure 5. The run of the differential abundance $\Delta[\mathrm{Mg} / \mathrm{Fe}]$ (top panel) and $\Delta[\mathrm{Zr} / \mathrm{Fe}]$ (bottom panel) with $\Delta[\mathrm{La} / \mathrm{Fe}]$ for the stars in the Hyades open cluster and supercluster analysed by Pompéia et al. (2011). Abundance ratios are referenced to the values obtained for the Hyades cluster member HD 26756. The symbols (see Figure 4) distinguish certain and possible Hyades open cluster members, possible evaporated cluster stars among the supercluster's members and other members of the supercluster. 


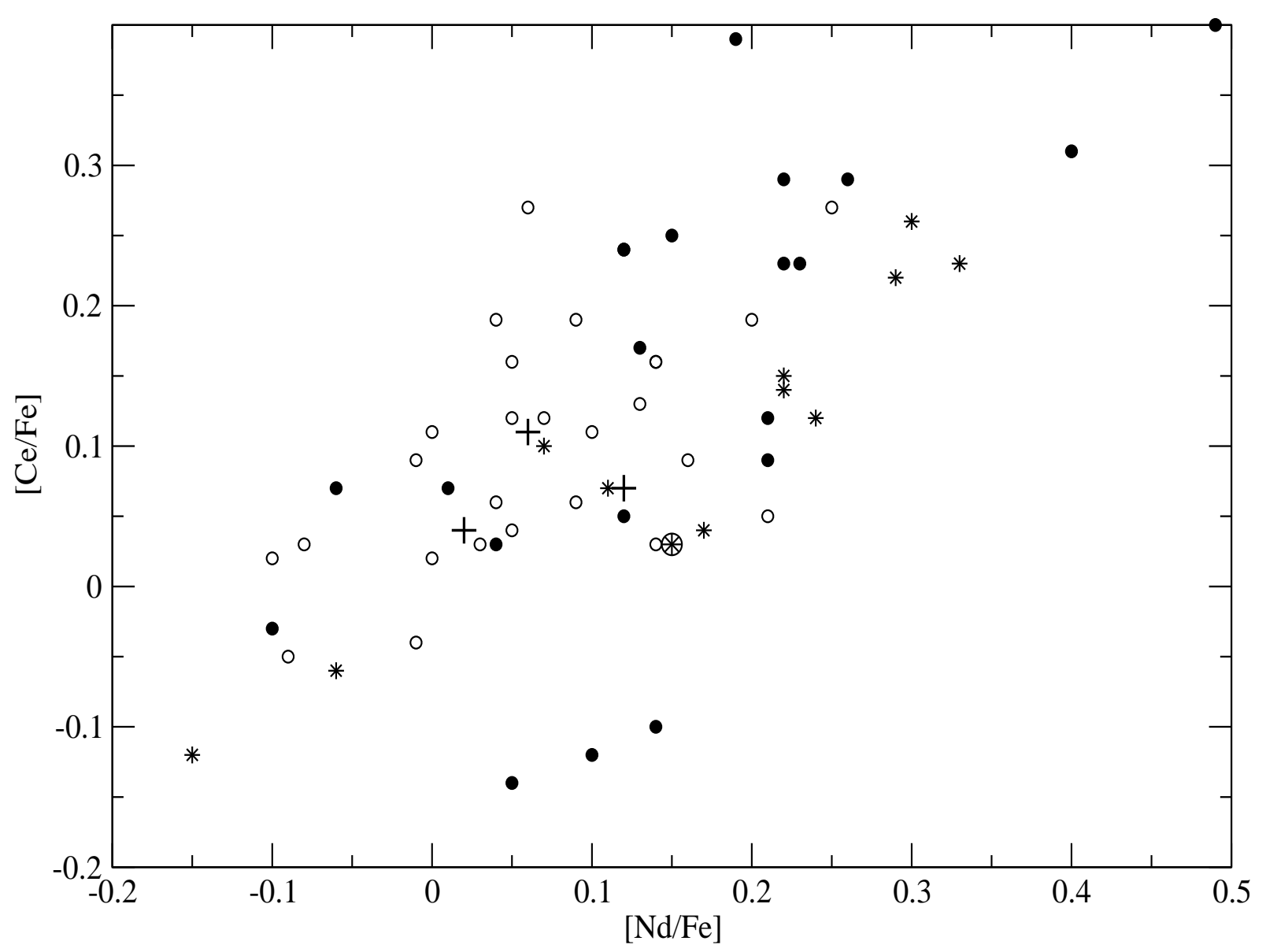

Figure 6. The run of $[\mathrm{Ce} / \mathrm{Fe}]$ with $[\mathrm{Nd} / \mathrm{Fe}]$ for Hyades open cluster and supercluster stars analysed by Tabernero et al. (2012). Hyades open cluster main sequence stars are represented by + and the cluster's giant $\epsilon$ Tau by an encircled star symbol. Other giant stars are represented by star symbols. Supercluster main sequence stars satisfying the $[\mathrm{Fe} / \mathrm{H}]$ limits $-0.04 \leqslant[\mathrm{Fe} / \mathrm{H}] \leqslant+0.16(\mathrm{i} . e$. , potential evaporated stars from the Hyades open cluster) are shown by open circles. Supercluster stars with $[\mathrm{Fe} / \mathrm{H}]$ outside the above limits are represented by filled circles. 


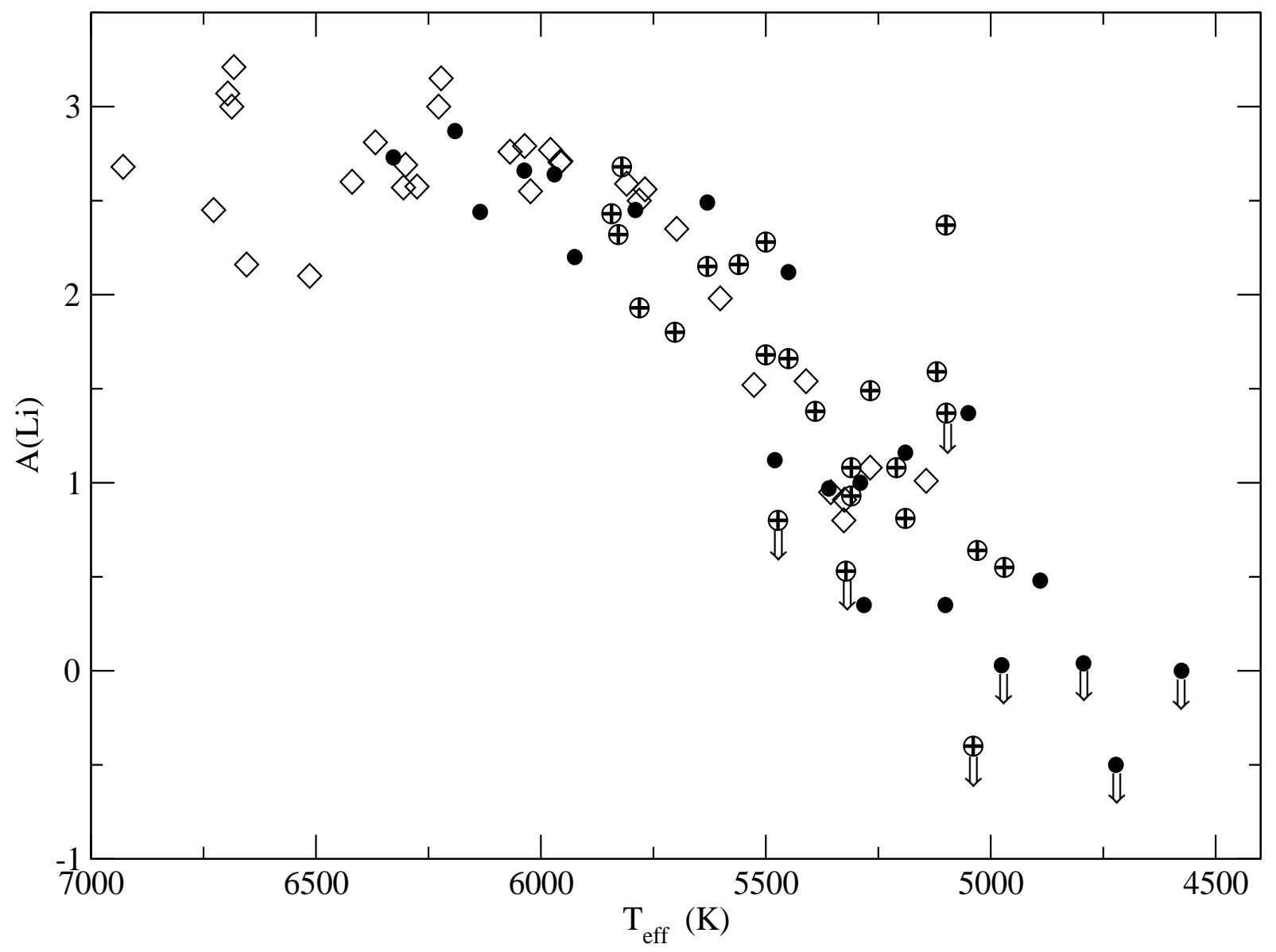

Figure 7. The Lithium abundance $\log \epsilon(\mathrm{Li})$ trend with effective temperature $\mathrm{T}_{\text {eff }}$ for Hyades supercluster main sequence candidates selected by Tabernero et al. (2012). Stars satisfying their $[\mathrm{Fe} / \mathrm{H}]$ criteria for prior membership of the Hyades open cluster are represented by a encircled + . Stars failing the $[\mathrm{Fe} / \mathrm{H}]$ criteria are shown as filled circles. The diamond symbol shows the run of the Li abundance obtained by Cummings et al. (2017) from main sequence members of the Hyades open cluster; Hyades and Praesepe open clusters show essentially the same decline with effective temperature. The down arrows mark upper limits on the Li abundance 


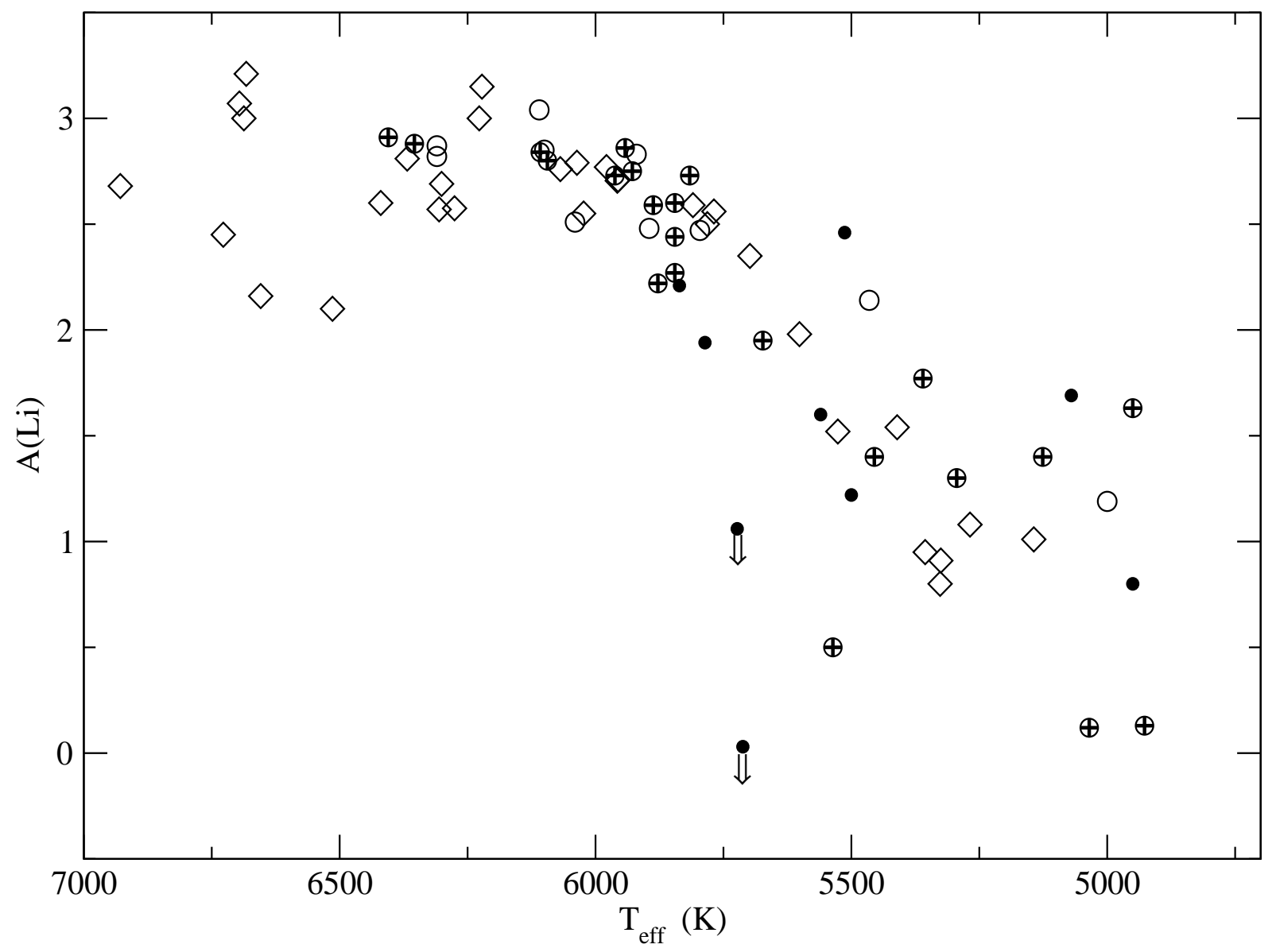

Figure 8. The Lithium abundance $\log \epsilon(\mathrm{Li})$ trend with effective temperature $\mathrm{T}_{\text {eff }}$ for Sirius supercluster main sequence candidates analysed by Tabernero et al. (2017). Stars satisfying their $[\mathrm{Fe} / \mathrm{H}]$ criterion to have been evaporated from the U Ma open cluster are represented by a encircled + . Stars failing that $[\mathrm{Fe} / \mathrm{H}]$ criterion are shown as filled circles. Additional stars assigned to the supercluster with a $\mathrm{Li}$ abundance determination by Ammler-von Eiff \& Guenther (2009) are shown by open circles. Abundances for Hyades open cluster main sequence stars (diamond) are taken from Cummings et al. (2017). The down arrows mark upper limits on the Li abundance 
Table 1: Derived Atmospheric Parameters of giants from the Hyades supercluster.

\begin{tabular}{|c|c|c|c|c|c|c|c|}
\hline Star & $\begin{array}{c}\left(T_{\text {eff }}\right)_{V-K} \\
\mathrm{~K}\end{array}$ & $\begin{array}{c}\left(T_{\text {eff }}\right)_{\text {spec }} \\
\mathrm{K}\end{array}$ & $\begin{array}{c}\log g \text { (phot) } \\
\mathrm{cm} \mathrm{s}^{-2}\end{array}$ & $\begin{array}{c}\log g(\mathrm{spec}) \\
\mathrm{cm} \mathrm{s}^{-2}\end{array}$ & $\begin{array}{c}\xi_{t} \\
\mathrm{~km} \mathrm{~s}^{-1}\end{array}$ & $\begin{array}{c}{[\mathrm{M} / \mathrm{H}]_{\text {model }}} \\
\text { dex }\end{array}$ & $\begin{array}{l}\text { Age } \\
\text { Gyr }\end{array}$ \\
\hline HIP1421 & 5257 & 5040 & 2.810 .04 & 2.95 & 1.52 & +0.05 & 1.10 .2 \\
\hline HIP2320 & 4735 & 4870 & 2.840 .04 & 2.78 & 1.60 & +0.25 & 1.40 .2 \\
\hline HIP6939 & 4651 & 4570 & 2.450 .08 & 2.35 & 1.65 & +0.23 & 4.82 .5 \\
\hline HIP13887 & 4898 & 5020 & 2.840 .04 & 2.80 & 1.49 & +0.10 & $\begin{array}{ll}1.2 & 0.2\end{array}$ \\
\hline HIP17752 & 4689 & 4740 & 2.740 .15 & 2.52 & 1.46 & -0.19 & 5.82 .4 \\
\hline HIP18565 & 5124 & 4740 & 2.540 .08 & 2.60 & 1.47 & +0.04 & 1.00 .4 \\
\hline HIP19222 & 5030 & 4940 & 2.850 .03 & 2.91 & 1.42 & +0.19 & 1.30 .2 \\
\hline HIP19641 & 5131 & 4760 & 2.570 .09 & 2.57 & 1.60 & +0.07 & 1.91 .2 \\
\hline HIP24633 & 5208 & 4930 & 2.600 .08 & 2.65 & 1.56 & +0.12 & $\begin{array}{ll}0.6 & 0.2\end{array}$ \\
\hline HIP35317 & 4944 & 4890 & 2.680 .07 & 2.67 & 1.50 & +0.01 & 1.10 .4 \\
\hline HIP34043 & 4959 & 4850 & 2.740 .08 & 2.54 & 1.69 & +0.15 & 1.20 .4 \\
\hline HIP36739 & 4847 & 4750 & 2.430 .09 & 2.41 & 1.52 & -0.04 & 0.70 .3 \\
\hline HIP 41172 & 4932 & 4870 & 2.820 .05 & 2.84 & 1.49 & +0.22 & 1.30 .2 \\
\hline HIP 49163 & 4759 & 4790 & 2.530 .10 & 2.58 & 1.50 & -0.04 & 3.01 .6 \\
\hline HIP51091 & 4697 & 4720 & 2.700 .12 & 2.55 & 1.60 & +0.18 & 1.90 .8 \\
\hline HIP51224 & 4745 & 4750 & 2.440 .07 & 2.28 & 1.53 & -0.18 & 4.02 .3 \\
\hline HIP 56756 & 4794 & 4740 & 2.610 .10 & 2.58 & 1.59 & +0.16 & 2.01 .0 \\
\hline HIP62405 & 5052 & 4980 & 2.850 .06 & 2.78 & 1.34 & +0.07 & $\begin{array}{ll}1.2 & 0.2\end{array}$ \\
\hline HIP65366 & 4725 & 4800 & 2.490 .09 & 2.55 & 1.53 & -0.10 & 1.91 .7 \\
\hline HIP74080 & 4911 & 4970 & 2.890 .04 & 2.88 & 1.41 & +0.07 & 1.30 .2 \\
\hline HIP79164 & 4674 & 4770 & 2.560 .10 & 2.58 & 1.54 & +0.05 & 2.61 .3 \\
\hline HIP79647 & 4657 & 4710 & 2.490 .09 & 2.58 & 1.42 & +0.03 & 3.52 .0 \\
\hline HIP79867 & 4459 & 4360 & 2.040 .08 & 2.07 & 1.71 & +0.18 & 2.00 .8 \\
\hline HIP80656 & 4795 & 4850 & 2.660 .08 & 2.63 & 1.51 & +0.06 & 1.00 .5 \\
\hline HIP80839 & 4926 & 4950 & 2.750 .05 & 2.70 & 1.50 & +0.17 & 1.00 .2 \\
\hline HIP82219 & 4949 & 4950 & 2.830 .06 & 2.72 & 1.43 & +0.02 & 1.30 .2 \\
\hline HIP83289 & 4904 & 4920 & 2.840 .03 & 2.97 & 1.45 & +0.21 & 1.30 .2 \\
\hline HIP85160 & 4958 & 4950 & 2.690 .06 & 2.77 & 1.43 & +0.03 & 0.80 .2 \\
\hline HIP88204 & 4494 & 4670 & $2.66 \quad 0.14$ & 2.56 & 1.63 & +0.16 & 2.61 .5 \\
\hline HIP93589 & 4862 & 4710 & 2.490 .11 & 2.44 & 1.51 & -0.03 & 4.92 .9 \\
\hline HIP113084 & 4892 & 4650 & 2.490 .08 & 2.54 & 1.63 & +0.15 & 3.71 .8 \\
\hline HIP113635 & 4915 & 4590 & 2.270 .10 & 2.28 & 1.54 & -0.06 & 2.01 .2 \\
\hline HIP114565 & 4946 & 4970 & 2.770 .06 & 2.84 & 1.53 & +0.20 & 1.00 .2 \\
\hline HIP117954 & 4456 & 4530 & 2.400 .07 & 2.28 & 1.58 & +0.05 & 5.62 .7 \\
\hline
\end{tabular}


Table 2: Derived Atmospheric Parameters of giants from the Sirius supercluster.

\begin{tabular}{lccccccc}
\hline Star & $\begin{array}{c}\left(T_{\text {eff }}\right)_{V-K} \\
\mathrm{~K}\end{array}$ & $\begin{array}{c}\left(T_{\text {eff }}\right)_{\text {spec }} \\
\mathrm{K}\end{array}$ & $\begin{array}{c}\log g(\mathrm{phot}) \\
\mathrm{cm} \mathrm{s}^{-2}\end{array}$ & $\begin{array}{c}\log g(\mathrm{spec}) \\
\mathrm{cm} \mathrm{s}^{-2}\end{array}$ & $\begin{array}{c}\xi_{t} \\
\mathrm{~km} \mathrm{~s}^{-1}\end{array}$ & $\begin{array}{c}{[\mathrm{M} / \mathrm{H}]_{\text {model }}} \\
\mathrm{dex}\end{array}$ & $\begin{array}{c}\text { Age } \\
\mathrm{Gyr}\end{array}$ \\
\hline \hline HIP7668 & 4276 & 4210 & 1.680 .14 & 1.55 & 1.82 & -0.08 & 3.02 .6 \\
HIP26711 & 4512 & 5020 & 2.640 .09 & 2.52 & 1.70 & +0.14 & 0.60 .2 \\
HIP28556 & 4563 & 4510 & 2.300 .12 & 2.21 & 1.56 & +0.07 & 3.02 .3 \\
HIP35431 & 5081 & 5100 & 2.720 .08 & 2.70 & 1.43 & +0.01 & 0.80 .2 \\
HIP37030 & 4410 & 4490 & 2.010 .16 & 1.98 & 1.65 & -0.09 & 1.11 .0 \\
HIP47719 & 4450 & 4550 & 2.260 .11 & 2.28 & 1.59 & +0.10 & 1.41 .1 \\
HIP49103 & 4357 & 4360 & 2.020 .11 & 1.98 & 1.67 & +0.04 & 3.11 .6 \\
HIP51825 & 5010 & 5120 & 2.860 .18 & 2.84 & 1.52 & +0.15 & 0.70 .3 \\
HIP52926 & 4813 & 4920 & 2.700 .09 & 2.72 & 1.44 & +0.05 & 0.90 .3 \\
HIP53710 & 4220 & 4310 & 2.811 .22 & 2.00 & 1.65 & +0.06 & 4.03 .5 \\
HIP53876 & 5031 & 5140 & 2.780 .09 & 2.89 & 1.42 & +0.10 & 0.70 .2 \\
HIP66567 & 4755 & 4820 & 2.830 .12 & 2.90 & 1.30 & +0.01 & 2.11 .0 \\
HIP67021 & 4228 & 4290 & 1.850 .10 & 1.83 & 1.63 & -0.02 & 3.32 .1 \\
HIP67364 & 4510 & 4570 & 2.380 .13 & 2.35 & 1.53 & +0.07 & 3.53 .2 \\
HIP68590 & 4758 & 4840 & 2.710 .23 & 2.60 & 1.51 & -0.03 & 1.91 .4 \\
HIP68737 & 4751 & 4810 & 2.750 .14 & 2.60 & 1.51 & +0.08 & 1.70 .8 \\
HIP68828 & 4969 & 5070 & 2.740 .10 & 2.90 & 1.46 & +0.07 & 0.80 .3 \\
HIP68935 & 4710 & 4800 & 2.790 .11 & 2.68 & 1.32 & -0.11 & 3.51 .5 \\
HIP77401 & 4208 & 4250 & 1.870 .11 & 1.76 & 1.68 & +0.03 & 4.32 .5 \\
HIP79326 & 4294 & 4330 & 1.860 .13 & 1.82 & 1.50 & -0.22 & 4.82 .9 \\
HIP80211 & 4870 & 4960 & 2.790 .10 & 2.70 & 1.49 & -0.07 & 1.20 .4 \\
HIP117938 & 4297 & 4390 & 2.070 .12 & 2.06 & 1.59 & +0.08 & 2.21 .2 \\
& & & & & & & \\
\hline
\end{tabular}


Table 3: The line data adopted for the current study.

\begin{tabular}{|c|c|c|c|c|c|c|c|c|}
\hline Species & $\begin{array}{c}\text { Wavelength } \\
(\AA) \\
\end{array}$ & LEP & $\overline{\log g f}$ & $\begin{array}{l}\mathrm{W}_{\lambda \odot} \\
(\mathrm{m \AA}) \\
\end{array}$ & $\begin{array}{c}\log \varepsilon_{\odot} \\
\operatorname{dex}\end{array}$ & $\begin{array}{c}\log \varepsilon \pm \sigma \\
\text { current study } \\
\operatorname{dex} \\
\end{array}$ & $\begin{array}{c}\log \varepsilon \pm \sigma \\
\text { Asplund et al.(2009) } \\
\operatorname{dex}\end{array}$ & $\begin{array}{c}\text { Difference } \\
\text { current - Asplund } \\
\text { dex } \\
\end{array}$ \\
\hline \multirow[t]{2}{*}{$\mathrm{NaI}$} & 6154.230 & 2.100 & -1.550 & 36.6 & 6.26 & $6.27 \pm 0.02$ & $6.24 \pm 0.04$ & +0.03 \\
\hline & 6160.750 & 2.100 & -1.250 & 56.5 & 6.28 & & & \\
\hline \multirow[t]{4}{*}{$\mathrm{Mg} \mathrm{I}$} & 5711.090 & 4.340 & -1.730 & 104.1 & 7.57 & $7.60 \pm 0.03$ & $7.60 \pm 0.04$ & 0.00 \\
\hline & 6318.720 & 5.110 & -1.950 & 44.5 & 7.57 & & & \\
\hline & 6319.240 & 5.110 & -2.320 & 27.4 & 7.63 & & & \\
\hline & 7657.610 & 5.110 & -1.280 & 98.5 & 7.62 & & & \\
\hline \multirow[t]{4}{*}{$\mathrm{Al} \mathrm{I}$} & 6696.020 & 3.140 & -1.480 & 36.9 & 6.38 & $6.37 \pm 0.03$ & $6.45 \pm 0.03$ & -0.08 \\
\hline & 6698.670 & 3.140 & -1.780 & 20.8 & 6.34 & & & \\
\hline & 7835.310 & 4.020 & -0.690 & 41.1 & 6.41 & & & \\
\hline & 7836.130 & 4.020 & -0.450 & 55.0 & 6.36 & & & \\
\hline \multirow[t]{5}{*}{ Si I } & 5690.420 & 4.930 & -1.770 & 48.1 & 7.46 & $7.52 \pm 0.06$ & $7.51 \pm 0.03$ & +0.01 \\
\hline & 5701.100 & 4.930 & -1.950 & 37.9 & 7.46 & & & \\
\hline & 5772.150 & 5.080 & -1.650 & 52.3 & 7.55 & & & \\
\hline & 6142.490 & 5.620 & -1.540 & 33.3 & 7.57 & & & \\
\hline & 6145.020 & 5.610 & -1.479 & 37.6 & 7.58 & & & \\
\hline \multirow[t]{8}{*}{$\mathrm{Ca} \mathrm{I}$} & 5260.390 & 2.520 & -1.720 & 32.1 & 6.25 & $6.29 \pm 0.04$ & $6.34 \pm 0.04$ & -0.05 \\
\hline & 5867.560 & 2.930 & -1.570 & 22.6 & 6.25 & & & \\
\hline & 6166.440 & 2.520 & -1.140 & 69.1 & 6.34 & & & \\
\hline & 6169.040 & 2.520 & -0.800 & 90.3 & 6.36 & & & \\
\hline & 6169.560 & 2.530 & -0.480 & 108.7 & 6.32 & & & \\
\hline & 6455.600 & 2.520 & -1.340 & 55.9 & 6.29 & & & \\
\hline & 6471.660 & 2.530 & -0.690 & 90.6 & 6.25 & & & \\
\hline & 6499.650 & 2.520 & -0.820 & 84.7 & 6.27 & & & \\
\hline \multirow[t]{6}{*}{ Sc II } & 5357.200 & 1.510 & -2.110 & 4.8 & 3.13 & $3.16 \pm 0.05$ & $3.15 \pm 0.04$ & +0.01 \\
\hline & 5552.230 & 1.460 & -2.280 & 4.6 & 3.22 & & & \\
\hline & 5684.210 & 1.510 & -1.070 & 37.1 & 3.18 & & & \\
\hline & 6245.640 & 1.510 & -1.040 & 35.2 & 3.09 & & & \\
\hline & 6300.750 & 1.510 & -1.950 & 8.2 & 3.18 & & & \\
\hline & 6320.840 & 1.500 & -1.920 & 8.9 & 3.18 & & & \\
\hline \multirow[t]{9}{*}{ Ti I } & 5295.770 & 1.070 & -1.580 & 13.2 & 4.89 & $4.87 \pm 0.05$ & $4.95 \pm 0.05$ & -0.08 \\
\hline & 5490.150 & 1.460 & -0.880 & 22.0 & 4.84 & & & \\
\hline & 5702.660 & 2.290 & -0.590 & 7.3 & 4.78 & & & \\
\hline & 5716.440 & 2.300 & -0.720 & 5.9 & 4.82 & & & \\
\hline & 6092.790 & 1.890 & -1.320 & 4.1 & 4.835 & & & \\
\hline & 6303.750 & 1.440 & -1.510 & 8.8 & 4.93 & & & \\
\hline & 6312.230 & 1.460 & -1.500 & 8.1 & 4.90 & & & \\
\hline & 6599.100 & 0.900 & -2.030 & 9.3 & 4.92 & & & \\
\hline & 7357.730 & 1.440 & -1.070 & 22.2 & 4.92 & & & \\
\hline \multirow[t]{4}{*}{ Ti II } & 4583.410 & 1.170 & -2.870 & 33.0 & 5.03 & $5.00 \pm 0.04$ & $4.95 \pm 0.05$ & +0.05 \\
\hline & 4708.660 & 1.240 & -2.370 & 53.3 & 5.04 & & & \\
\hline & 5336.780 & 1.580 & -1.630 & 71.7 & 4.99 & & & \\
\hline & 5418.770 & 1.580 & -2.110 & 48.5 & 4.96 & & & \\
\hline \multirow[t]{6}{*}{ V I } & 6039.730 & 1.060 & -0.650 & 12.3 & 3.87 & $3.84 \pm 0.02$ & $3.93 \pm 0.08$ & -0.09 \\
\hline & 6081.440 & 1.050 & -0.580 & 13.0 & 3.80 & & & \\
\hline & 6090.210 & 1.080 & -0.060 & 32.3 & 3.83 & & & \\
\hline & 6119.530 & 1.060 & -0.320 & 21.0 & 3.82 & & & \\
\hline & 6135.360 & 1.050 & -0.750 & 10.2 & 3.85 & & & \\
\hline & 6274.650 & 0.270 & -1.670 & 6.9 & 3.80 & & & \\
\hline \multirow[t]{7}{*}{ Cr I } & 5287.200 & 3.440 & -0.890 & 11.3 & 5.61 & $5.60 \pm 0.05$ & $5.64 \pm 0.04$ & -0.04 \\
\hline & 5300.740 & 0.980 & -2.080 & 59.3 & 5.59 & & & \\
\hline & 5304.180 & 3.460 & -0.680 & 16.0 & 5.60 & & & \\
\hline & 5628.620 & 3.420 & -0.760 & 14.7 & 5.58 & & & \\
\hline & 5781.160 & 3.010 & -1.000 & 16.7 & 5.50 & & & \\
\hline & 6882.480 & 3.440 & -0.380 & 32.4 & 5.65 & & & \\
\hline & 6883.000 & 3.440 & -0.420 & 30.5 & 5.65 & & & \\
\hline \multirow[t]{2}{*}{ Mn I } & 4671.690 & 2.890 & -1.660 & 14.8 & 5.42 & $5.40 \pm 0.02$ & $5.43 \pm 0.04$ & -0.03 \\
\hline & 4739.110 & 2.940 & -0.600 & 60.7 & 5.37 & & & \\
\hline
\end{tabular}




\begin{tabular}{|c|c|c|c|c|c|c|c|c|}
\hline \multirow[b]{2}{*}{$\mathrm{Fe} I$} & 5004.890 & 2.920 & -1.640 & 14.0 & 5.40 & \multirow[b]{2}{*}{$7.43 \pm 0.05$} & \multirow[b]{2}{*}{$7.50 \pm 0.04$} & \multirow[b]{2}{*}{-0.07} \\
\hline & 5198.720 & 2.220 & -2.130 & 94.1 & 7.37 & & & \\
\hline & 5253.020 & 2.280 & -3.940 & 18.5 & 7.53 & & & \\
\hline & 5295.310 & 4.420 & -1.590 & 29.1 & 7.51 & & & \\
\hline & 5322.030 & 2.280 & -2.800 & 59.5 & 7.32 & & & \\
\hline & 5358.120 & 3.300 & -3.162 & 9.5 & 7.39 & & & \\
\hline & 5379.570 & 3.690 & -1.510 & 60.5 & 7.41 & & & \\
\hline & 5386.330 & 4.150 & -1.670 & 32.1 & 7.41 & & & \\
\hline & 5441.340 & 4.310 & -1.630 & 30.3 & 7.47 & & & \\
\hline & 5638.260 & 4.220 & -0.770 & 75.8 & 7.45 & & & \\
\hline & 5661.350 & 4.280 & -1.756 & 21.7 & 7.35 & & & \\
\hline & 5679.020 & 4.652 & -0.750 & 58.2 & 7.47 & & & \\
\hline & 5701.550 & 2.560 & -2.220 & 83.0 & 7.50 & & & \\
\hline & 5705.460 & 4.301 & -1.355 & 37.7 & 7.34 & & & \\
\hline & 5731.760 & 4.260 & -1.200 & 56.5 & 7.53 & & & \\
\hline & 5778.450 & 2.588 & -3.440 & 22.2 & 7.40 & & & \\
\hline & 5793.910 & 4.220 & -1.619 & 33.2 & 7.43 & & & \\
\hline & 5849.690 & 3.695 & -2.930 & 6.6 & 7.34 & & & \\
\hline & 5855.080 & 4.608 & -1.478 & 20.9 & 7.35 & & & \\
\hline & 5856.100 & 4.294 & -1.558 & 32.5 & 7.42 & & & \\
\hline & 5858.790 & 4.220 & -2.180 & 12.5 & 7.41 & & & \\
\hline & 5859.600 & 4.550 & -0.608 & 69.3 & 7.44 & & & \\
\hline & 5905.670 & 4.650 & -0.690 & 56.8 & 7.38 & & & \\
\hline & 5927.790 & 4.650 & -0.990 & 41.5 & 7.38 & & & \\
\hline & 5929.680 & 4.550 & -1.310 & 39.5 & 7.56 & & & \\
\hline & 6003.010 & 3.880 & -1.060 & 81.7 & 7.53 & & & \\
\hline & 6015.250 & 2.220 & -4.680 & 4.4 & 7.45 & & & \\
\hline & 6027.050 & 4.076 & -1.090 & 63.2 & 7.37 & & & \\
\hline & 6056.000 & 4.730 & -0.400 & 70.6 & 7.41 & & & \\
\hline & 6065.490 & 2.610 & -1.530 & 113.7 & 7.37 & & & \\
\hline & 6079.010 & 4.650 & -1.020 & 44.9 & 7.47 & & & \\
\hline & 6093.640 & 4.607 & -1.300 & 30.2 & 7.40 & & & \\
\hline & 6096.660 & 3.984 & -1.810 & 36.9 & 7.46 & & & \\
\hline & 6120.250 & 0.920 & -5.970 & 5.5 & 7.54 & & & \\
\hline & 6137.000 & 2.200 & -2.950 & 63.7 & 7.41 & & & \\
\hline & 6151.620 & 2.176 & -3.282 & 48.7 & 7.41 & & & \\
\hline & 6159.380 & 4.610 & -1.830 & 12.6 & 7.42 & & & \\
\hline & 6165.360 & 4.143 & -1.460 & 43.9 & 7.41 & & & \\
\hline & 6173.340 & 2.220 & -2.880 & 66.1 & 7.41 & & & \\
\hline & 6180.210 & 2.730 & -2.650 & 52.9 & 7.41 & & & \\
\hline & 6187.990 & 3.940 & -1.620 & 46.1 & 7.42 & & & \\
\hline & 6213.440 & 2.220 & -2.480 & 81.0 & 7.33 & & & \\
\hline & 6219.290 & 2.200 & -2.430 & 87.1 & 7.39 & & & \\
\hline & 6240.650 & 2.223 & -3.287 & 47.8 & 7.43 & & & \\
\hline & 6252.560 & 2.400 & -1.690 & 116.6 & 7.36 & & & \\
\hline & 6265.140 & 2.180 & -2.550 & 83.9 & 7.41 & & & \\
\hline & 6270.230 & 2.858 & -2.540 & 51.6 & 7.40 & & & \\
\hline & 6271.280 & 3.330 & -2.703 & 23.8 & 7.41 & & & \\
\hline & 6322.700 & 2.590 & -2.430 & 73.3 & 7.47 & & & \\
\hline & 6335.350 & 2.200 & -2.350 & 94.9 & 7.45 & & & \\
\hline & 6344.150 & 2.430 & -2.920 & 56.5 & 7.45 & & & \\
\hline & 6392.540 & 2.280 & -4.030 & 17.3 & 7.52 & & & \\
\hline & 6436.410 & 4.186 & -2.360 & 10.0 & 7.42 & & & \\
\hline & 6475.630 & 2.560 & -2.940 & 53.0 & 7.52 & & & \\
\hline & 6481.880 & 2.280 & -2.980 & 61.6 & 7.45 & & & \\
\hline & 6498.940 & 0.960 & -4.690 & 44.0 & 7.46 & & & \\
\hline & 6518.370 & 2.830 & -2.450 & 56.2 & 7.36 & & & \\
\hline & 6575.020 & 2.590 & -2.710 & 59.1 & 7.44 & & & \\
\hline & 6581.210 & 1.480 & -4.680 & 20.8 & 7.46 & & & \\
\hline & 6591.330 & 4.593 & -1.950 & 10.4 & 7.41 & & & \\
\hline & 6608.030 & 2.280 & -4.030 & 17.3 & 7.50 & & & \\
\hline & 6609.110 & 2.560 & -2.690 & 63.6 & 7.48 & & & \\
\hline
\end{tabular}




\begin{tabular}{|c|c|c|c|c|c|c|c|}
\hline 6699.140 & 4.590 & -2.100 & 7.9 & 7.42 & & & \\
\hline 6703.570 & 2.759 & -3.023 & 36.1 & 7.43 & & & \\
\hline 6705.100 & 4.607 & -0.980 & 45.4 & 7.38 & & & \\
\hline 6710.320 & 1.490 & -4.870 & 15.0 & 7.47 & & & \\
\hline 6713.750 & 4.795 & -1.400 & 20.8 & 7.42 & & & \\
\hline 6725.360 & 4.103 & -2.167 & 17.0 & 7.41 & & & \\
\hline 6726.670 & 4.607 & -1.030 & 46.0 & 7.44 & & & \\
\hline 6733.150 & 4.638 & -1.400 & 25.9 & 7.41 & & & \\
\hline 6739.520 & 1.560 & -4.794 & 11.5 & 7.33 & & & \\
\hline 6750.160 & 2.420 & -2.620 & 72.5 & 7.44 & & & \\
\hline 6793.260 & 4.076 & -2.326 & 12.4 & 7.38 & & & \\
\hline 6810.260 & 4.607 & -0.986 & 48.6 & 7.44 & & & \\
\hline 6828.590 & 4.640 & -0.820 & 54.6 & 7.43 & & & \\
\hline 6837.010 & 4.590 & -1.687 & 17.6 & 7.42 & & & \\
\hline 6842.690 & 4.640 & -1.220 & 39.1 & 7.52 & & & \\
\hline 6843.660 & 4.550 & -0.830 & 59.5 & 7.44 & & & \\
\hline 6857.250 & 4.076 & -2.038 & 22.3 & 7.41 & & & \\
\hline 6911.510 & 2.420 & -4.040 & 13.0 & 7.49 & & & \\
\hline 6971.940 & 3.020 & -3.340 & 12.6 & 7.36 & & & \\
\hline 6999.880 & 4.100 & -1.460 & 53.8 & 7.53 & & & \\
\hline 7022.950 & 4.190 & -1.150 & 63.6 & 7.50 & & & \\
\hline 7132.990 & 4.080 & -1.650 & 42.1 & 7.46 & & & \\
\hline 7751.120 & 4.990 & -0.730 & 45.3 & 7.44 & & & \\
\hline 7802.510 & 5.080 & -1.310 & 15.4 & 7.40 & & & \\
\hline 7807.920 & 4.990 & -0.509 & 58.8 & 7.47 & & & \\
\hline 8757.120 & 2.850 & -2.030 & 92.1 & 7.45 & & & \\
\hline 8804.520 & 2.280 & -3.230 & 58.2 & 7.45 & & & \\
\hline 8838.330 & 2.860 & -1.980 & 94.7 & 7.44 & & & \\
\hline 5197.570 & 3.230 & -2.220 & 78.1 & 7.37 & $7.44 \pm 0.05$ & $7.50 \pm 0.04$ & -0.06 \\
\hline 5234.620 & 3.221 & -2.180 & 83.3 & 7.43 & & & \\
\hline 5256.920 & 2.890 & -4.060 & 20.1 & 7.44 & & & \\
\hline 5264.800 & 3.230 & -3.130 & 47.9 & 7.55 & & & \\
\hline 5414.070 & 3.221 & -3.580 & 27.5 & 7.48 & & & \\
\hline 5425.260 & 3.200 & -3.220 & 41.3 & 7.45 & & & \\
\hline 5534.840 & 3.250 & -2.750 & 56.9 & 7.40 & & & \\
\hline 6149.250 & 3.889 & -2.630 & 35.7 & 7.37 & & & \\
\hline 6247.560 & 3.892 & -2.271 & 52.8 & 7.42 & & & \\
\hline 6369.460 & 2.891 & -4.110 & 19.4 & 7.45 & & & \\
\hline 6456.390 & 3.903 & -2.065 & 62.3 & 7.44 & & & \\
\hline 6516.080 & 2.891 & -3.310 & 52.6 & 7.48 & & & \\
\hline 5280.630 & 3.630 & -0.030 & 20.3 & 4.82 & $4.81 \pm 0.02$ & $4.99 \pm 0.07$ & -0.18 \\
\hline 5352.040 & 3.580 & 0.060 & 25.1 & 4.79 & & & \\
\hline 5647.230 & 2.280 & -1.560 & 13.9 & 4.83 & & & \\
\hline 6455.000 & 3.630 & -0.250 & 14.8 & 4.82 & & & \\
\hline 5088.960 & 3.678 & -1.240 & 28.2 & 6.16 & $6.23 \pm 0.05$ & $6.22 \pm 0.04$ & +0.01 \\
\hline 5094.420 & 3.833 & -1.074 & 30.4 & 6.19 & & & \\
\hline 5115.400 & 3.834 & -0.281 & 75.2 & 6.33 & & & \\
\hline 6111.080 & 4.088 & -0.808 & 33.6 & 6.21 & & & \\
\hline 6130.140 & 4.266 & -0.938 & 21.6 & 6.21 & & & \\
\hline 6175.370 & 4.089 & -0.550 & 47.7 & 6.25 & & & \\
\hline 6176.800 & 4.090 & -0.260 & 62.0 & 6.24 & & & \\
\hline 6177.250 & 1.826 & -3.508 & 14.1 & 6.17 & & & \\
\hline 6772.320 & 3.658 & -0.972 & 48.0 & 6.24 & & & \\
\hline 7797.590 & 3.900 & -0.348 & 75.3 & 6.31 & & & \\
\hline 7826.770 & 3.700 & -1.840 & 12.5 & 6.20 & & & \\
\hline 4810.540 & 4.080 & -0.170 & 71.6 & 4.52 & $4.52 \pm 0.01$ & $4.56 \pm 0.05$ & -0.04 \\
\hline 6362.350 & 5.790 & 0.140 & 21.2 & 4.53 & & & \\
\hline 5200.390 & 0.990 & -0.570 & 36.3 & 2.12 & $2.17 \pm 0.06$ & $2.21 \pm 0.05$ & -0.04 \\
\hline 5289.820 & 1.030 & -1.850 & 3.8 & 2.16 & & & \\
\hline 5402.760 & 1.840 & -0.620 & 11.6 & 2.24 & & & \\
\hline 4883.670 & 1.080 & 0.070 & 56.5 & 2.11 & & & \\
\hline 4982.110 & 1.030 & -1.290 & 13.4 & 2.23 & & & \\
\hline
\end{tabular}




\begin{tabular}{|c|c|c|c|c|c|c|c|c|}
\hline Zr I & $\begin{array}{l}4739.470 \\
6143.210\end{array}$ & $\begin{array}{l}0.650 \\
0.070\end{array}$ & $\begin{array}{c}0.230 \\
-1.100\end{array}$ & $\begin{array}{l}6.7 \\
2.3\end{array}$ & $\begin{array}{l}2.51 \\
2.68\end{array}$ & $2.60 \pm 0.12$ & $2.58 \pm 0.04$ & +0.02 \\
\hline \multirow[t]{2}{*}{ Ba II } & 5853.680 & 0.604 & -1.000 & 60.3 & 2.14 & $2.14 \pm 0.01$ & $2.18 \pm 0.09$ & -0.04 \\
\hline & 6141.730 & 0.704 & -0.032 & 109.1 & 2.14 & & & \\
\hline \multirow[t]{4}{*}{ La II } & 6390.480 & 0.320 & -1.410 & 2.7 & 1.09 & $1.19 \pm 0.09$ & $1.10 \pm 0.04$ & +0.09 \\
\hline & 4662.490 & 0.000 & -1.240 & 7.0 & 1.14 & & & \\
\hline & 5303.540 & 0.320 & -1.350 & 3.9 & 1.25 & & & \\
\hline & 6774.240 & 0.130 & -1.820 & 2.6 & 1.27 & & & \\
\hline \multirow[t]{3}{*}{ Nd II } & 5319.810 & 0.550 & -0.140 & 10.9 & 1.40 & $1.40 \pm 0.03$ & $1.42 \pm 0.04$ & -0.02 \\
\hline & 5092.790 & 0.380 & -0.610 & 5.5 & 1.37 & & & \\
\hline & 4989.920 & 0.630 & -0.310 & 6.9 & 1.44 & & & \\
\hline Eu II & 6645.130 & 1.380 & 0.204 & 5.4 & 0.56 & 0.53 & $0.52 \pm 0.04$ & +0.01 \\
\hline
\end{tabular}




\begin{tabular}{|c|c|c|c|c|c|c|c|c|}
\hline Star & {$[\mathrm{FeI} / \mathrm{H}] \sigma \mathrm{N}$} & {$[\mathrm{FeII} / \mathrm{H}] \sigma \mathrm{N}$} & {$[\mathrm{Na} / \mathrm{Fe}] \sigma \mathrm{N}$} & {$[\mathrm{Mg} / \mathrm{Fe}] \sigma \mathrm{N}$} & {$[\mathrm{Al} / \mathrm{Fe}] \sigma \mathrm{N}$} & {$[\mathrm{Si} / \mathrm{Fe}] \sigma \mathrm{N}$} & {$[\mathrm{Ca} / \mathrm{Fe}] \sigma \mathrm{N}$} & {$[\mathrm{Sc} / \mathrm{Fe}] \sigma \mathrm{N}$} \\
\hline HIP1421 & 0.050 .0883 & $\begin{array}{lll}0.03 & 0.05 & 12\end{array}$ & 0.270 .012 & 0.010 .054 & 0.070 .064 & 0.070 .065 & 0.050 .078 & -0.020 .046 \\
\hline HIP2320 & 0.260 .0883 & 0.240 .0811 & 0.280 .012 & 0.020 .054 & 0.140 .044 & 0.090 .065 & 0.000 .068 & -0.040 .046 \\
\hline HIP6939 & 0.230 .1078 & 0.230 .0610 & 0.330 .042 & 0.070 .034 & 0.240 .064 & 0.120 .095 & -0.090 .078 & -0.040 .056 \\
\hline HIP13887 & 0.110 .0786 & 0.110 .0411 & 0.180 .012 & -0.050 .053 & 0.050 .054 & 0.040 .075 & 0.040 .054 & -0.100 .046 \\
\hline HIP17752 & -0.180 .0685 & -0.190 .0611 & 0.080 .012 & -0.010 .044 & 0.100 .044 & 0.090 .085 & 0.060 .038 & 0.000 .036 \\
\hline HIP18565 & 0.040 .0885 & 0.040 .0712 & 0.170 .022 & -0.090 .054 & 0.050 .034 & 0.060 .065 & -0.020 .048 & -0.050 .036 \\
\hline HIP19222 & 0.190 .0885 & 0.190 .0712 & 0.170 .022 & -0.030 .054 & 0.130 .064 & 0.080 .075 & 0.000 .048 & -0.040 .046 \\
\hline HIP19641 & 0.060 .0885 & 0.060 .0712 & 0.130 .012 & 0.010 .044 & 0.120 .054 & 0.110 .075 & -0.010 .058 & -0.030 .046 \\
\hline HIP24633 & 0.120 .0787 & 0.100 .0611 & 0.370 .012 & -0.020 .013 & 0.100 .054 & 0.090 .075 & 0.020 .068 & -0.030 .045 \\
\hline HIP35317 & 0.020 .0785 & 0.010 .0411 & 0.140 .022 & -0.020 .013 & 0.090 .044 & 0.090 .075 & 0.050 .058 & -0.020 .025 \\
\hline HIP34043 & 0.160 .0985 & 0.140 .0710 & 0.310 .012 & -0.030 .024 & 0.100 .024 & 0.100 .065 & 0.000 .058 & -0.040 .046 \\
\hline HIP36739 & -0.040 .0786 & -0.040 .0712 & 0.150 .012 & -0.080 .024 & 0.100 .044 & 0.070 .075 & 0.030 .058 & -0.060 .046 \\
\hline HIP 41172 & 0.220 .0886 & 0.200 .0510 & 0.390 .012 & 0.000 .013 & 0.130 .054 & 0.100 .075 & 0.010 .068 & 0.020 .045 \\
\hline HIP49163 & -0.030 .0784 & -0.040 .0611 & 0.130 .012 & 0.040 .034 & 0.120 .024 & 0.100 .085 & 0.030 .058 & 0.010 .046 \\
\hline HIP51091 & 0.180 .0883 & 0.160 .0811 & 0.260 .012 & 0.010 .064 & 0.130 .014 & 0.100 .085 & -0.020 .068 & -0.000 .036 \\
\hline HIP51224 & -0.180 .0683 & -0.190 .0611 & 0.120 .032 & 0.120 .064 & 0.140 .024 & 0.150 .085 & 0.070 .068 & -0.000 .046 \\
\hline HIP 56756 & 0.150 .0883 & 0.140 .0711 & 0.220 .032 & -0.040 .044 & 0.120 .044 & 0.090 .095 & -0.020 .058 & -0.030 .026 \\
\hline HIP62405 & 0.070 .0984 & 0.060 .0812 & 0.250 .032 & 0.070 .054 & 0.110 .034 & 0.040 .085 & 0.090 .068 & -0.100 .046 \\
\hline HIP65366 & -0.100 .0785 & -0.120 .0412 & 0.080 .032 & 0.050 .024 & 0.150 .034 & 0.130 .065 & 0.040 .058 & 0.060 .036 \\
\hline HIP74080 & 0.070 .0785 & 0.060 .0610 & 0.210 .022 & -0.000 .043 & 0.050 .044 & 0.080 .055 & 0.020 .058 & -0.060 .046 \\
\hline HIP79164 & 0.050 .0784 & 0.030 .0611 & 0.160 .042 & 0.060 .073 & 0.110 .034 & 0.110 .075 & 0.010 .048 & 0.010 .046 \\
\hline HIP79647 & 0.040 .0782 & 0.030 .0411 & 0.160 .042 & 0.030 .032 & 0.100 .024 & 0.080 .065 & 0.000 .068 & -0.050 .026 \\
\hline HIP79867 & 0.170 .0977 & 0.160 .0610 & 0.310 .032 & 0.000 .033 & 0.160 .044 & 0.170 .065 & -0.110 .078 & -0.030 .066 \\
\hline HIP80656 & 0.060 .0685 & 0.030 .0511 & 0.090 .012 & 0.000 .062 & 0.060 .034 & 0.080 .055 & 0.010 .038 & -0.010 .046 \\
\hline HIP80839 & 0.170 .0784 & 0.150 .0712 & 0.290 .012 & -0.020 .012 & 0.070 .054 & 0.110 .075 & 0.010 .057 & -0.060 .046 \\
\hline HIP82219 & 0.030 .0684 & 0.010 .0612 & 0.130 .012 & -0.040 .012 & 0.030 .054 & 0.070 .065 & 0.030 .058 & -0.060 .046 \\
\hline HIP83289 & 0.220 .0785 & 0.200 .0610 & 0.260 .022 & 0.010 .063 & 0.060 .034 & 0.090 .055 & -0.010 .058 & -0.010 .046 \\
\hline HIP85160 & 0.020 .0784 & 0.010 .0611 & 0.140 .032 & -0.020 .032 & 0.060 .034 & 0.090 .075 & 0.040 .068 & -0.060 .056 \\
\hline HIP88204 & 0.160 .0882 & 0.150 .0711 & 0.270 .012 & 0.010 .001 & 0.150 .054 & 0.120 .065 & -0.060 .078 & 0.000 .055 \\
\hline HIP93589 & -0.030 .0785 & -0.030 .0511 & 0.090 .022 & 0.060 .012 & 0.110 .014 & 0.110 .065 & -0.010 .058 & -0.000 .045 \\
\hline HIP113084 & 0.150 .0985 & 0.150 .0610 & 0.200 .012 & 0.020 .043 & 0.120 .034 & 0.080 .035 & -0.080 .068 & -0.030 .056 \\
\hline HIP113635 & -0.060 .0783 & -0.070 .0712 & 0.090 .042 & 0.180 .014 & 0.240 .064 & 0.220 .075 & 0.040 .058 & -0.060 .036 \\
\hline HIP114565 & 0.210 .0884 & 0.190 .0712 & 0.230 .032 & -0.010 .044 & 0.060 .044 & 0.040 .075 & 0.020 .048 & -0.080 .046 \\
\hline HIP117954 & 0.060 .0983 & $\begin{array}{lll}0.05 & 0.08 & 12\end{array}$ & 0.210 .012 & 0.040 .044 & 0.130 .024 & 0.080 .065 & -0.060 .057 & -0.060 .036 \\
\hline
\end{tabular}


Table 5: Derived abundances of giants from the Hyades supercluster.

\begin{tabular}{|c|c|c|c|c|c|c|c|}
\hline Star & {$[\mathrm{TiI} / \mathrm{Fe}] \sigma \mathrm{N}$} & {$[\mathrm{TiII} / \mathrm{Fe}] \sigma \mathrm{N}$} & {$[\mathrm{V} / \mathrm{Fe}] \sigma \mathrm{N}$} & {$[\mathrm{Cr} / \mathrm{Fe}] \sigma \mathrm{N}$} & {$[\mathrm{Mn} / \mathrm{Fe}] \sigma \mathrm{N}$} & {$[\mathrm{Co} / \mathrm{Fe}] \sigma \mathrm{N}$} & {$[\mathrm{Ni} / \mathrm{Fe}] \sigma \mathrm{N}$} \\
\hline HIP1421 & 0.000 .058 & -0.040 .064 & -0.010 .045 & 0.020 .076 & -0.060 .033 & -0.020 .034 & -0.010 .0510 \\
\hline HIP2320 & 0.000 .078 & -0.090 .054 & -0.020 .065 & 0.040 .086 & -0.030 .033 & 0.020 .054 & 0.050 .0511 \\
\hline HIP6939 & -0.090 .097 & -0.200 .034 & -0.060 .045 & 0.000 .076 & -0.090 .103 & 0.080 .054 & 0.090 .0411 \\
\hline HIP13887 & -0.010 .088 & -0.060 .044 & -0.070 .025 & -0.040 .054 & -0.110 .033 & -0.100 .034 & $\begin{array}{lll}-0.00 & 0.0511\end{array}$ \\
\hline HIP17752 & 0.030 .058 & 0.020 .034 & -0.010 .035 & -0.040 .065 & -0.060 .033 & -0.030 .044 & $\begin{array}{lll}-0.02 & 0.0511\end{array}$ \\
\hline HIP18565 & -0.050 .078 & -0.050 .024 & -0.070 .045 & -0.010 .075 & -0.110 .043 & -0.010 .034 & $\begin{array}{lll}-0.01 & 0.0511\end{array}$ \\
\hline HIP19222 & -0.050 .048 & -0.070 .024 & -0.070 .035 & 0.000 .086 & -0.090 .033 & -0.020 .044 & 0.020 .0711 \\
\hline HIP19641 & -0.030 .068 & -0.090 .044 & -0.030 .035 & 0.020 .086 & -0.050 .063 & 0.000 .044 & 0.010 .0511 \\
\hline HIP24633 & -0.050 .058 & -0.020 .034 & -0.060 .035 & -0.010 .085 & -0.080 .013 & -0.010 .044 & 0.010 .0611 \\
\hline HIP35317 & -0.030 .048 & 0.000 .044 & -0.070 .025 & -0.000 .075 & -0.140 .083 & -0.060 .034 & $\begin{array}{lll}-0.02 & 0.0511\end{array}$ \\
\hline HIP34043 & -0.050 .078 & -0.090 .034 & -0.060 .045 & 0.020 .096 & -0.110 .043 & -0.050 .034 & 0.020 .0611 \\
\hline HIP36739 & -0.030 .068 & -0.070 .034 & -0.070 .045 & 0.000 .095 & -0.150 .023 & -0.060 .034 & $\begin{array}{lll}-0.03 & 0.07 & 11\end{array}$ \\
\hline HIP41172 & -0.010 .068 & -0.060 .034 & -0.020 .045 & 0.000 .075 & -0.090 .073 & 0.040 .034 & 0.080 .0611 \\
\hline HIP49163 & -0.020 .068 & 0.010 .044 & -0.010 .015 & 0.010 .066 & -0.100 .023 & 0.000 .044 & 0.010 .0511 \\
\hline HIP51091 & -0.040 .058 & -0.150 .084 & -0.040 .055 & 0.040 .095 & -0.090 .063 & 0.050 .044 & 0.050 .0511 \\
\hline HIP51224 & 0.030 .058 & -0.050 .054 & -0.040 .035 & -0.000 .075 & -0.120 .053 & 0.030 .044 & 0.010 .0511 \\
\hline HIP56756 & -0.050 .068 & -0.100 .044 & -0.050 .035 & 0.020 .085 & -0.070 .053 & 0.040 .044 & 0.060 .0511 \\
\hline HIP62405 & 0.040 .076 & -0.100 .074 & 0.050 .015 & 0.040 .106 & -0.080 .093 & -0.030 .064 & 0.020 .0611 \\
\hline HIP65366 & 0.020 .046 & 0.040 .054 & 0.010 .045 & 0.020 .066 & -0.070 .013 & 0.030 .034 & 0.020 .0511 \\
\hline HIP74080 & -0.040 .047 & -0.010 .054 & -0.050 .035 & 0.000 .056 & -0.090 .013 & -0.040 .044 & $\begin{array}{lll}-0.03 & 0.0511\end{array}$ \\
\hline HIP79164 & 0.010 .057 & -0.030 .064 & -0.040 .045 & 0.010 .076 & -0.050 .063 & 0.020 .034 & -0.000 .0511 \\
\hline HIP79647 & -0.040 .066 & -0.040 .044 & -0.060 .025 & 0.000 .066 & -0.060 .073 & -0.010 .044 & $\begin{array}{lll}-0.01 & 0.0511\end{array}$ \\
\hline HIP79867 & -0.020 .066 & -0.120 .034 & $-0.02 \quad 0.015$ & 0.040 .046 & -0.150 .083 & 0.100 .054 & $0.08 \quad 0.0511$ \\
\hline HIP80656 & -0.030 .056 & 0.010 .054 & -0.050 .035 & 0.000 .056 & -0.090 .023 & -0.030 .024 & $\begin{array}{lll}-0.01 & 0.0511\end{array}$ \\
\hline HIP80839 & -0.090 .065 & -0.100 .044 & -0.050 .035 & 0.030 .066 & -0.010 .113 & -0.010 .054 & 0.020 .0711 \\
\hline HIP82219 & -0.050 .066 & -0.030 .034 & -0.060 .035 & 0.000 .046 & -0.070 .083 & -0.040 .034 & $\begin{array}{lll}-0.03 & 0.0611\end{array}$ \\
\hline HIP83289 & -0.050 .066 & -0.090 .044 & -0.010 .035 & 0.010 .076 & -0.120 .043 & 0.000 .044 & 0.040 .0511 \\
\hline HIP85160 & -0.030 .056 & -0.080 .044 & -0.040 .035 & 0.010 .076 & -0.080 .022 & -0.040 .044 & $\begin{array}{lll}-0.05 & 0.0511\end{array}$ \\
\hline HIP88204 & -0.020 .077 & -0.100 .034 & 0.000 .035 & -0.000 .076 & -0.100 .083 & 0.090 .034 & 0.060 .0511 \\
\hline HIP93589 & -0.050 .056 & -0.060 .034 & -0.080 .025 & -0.040 .076 & -0.100 .043 & -0.010 .054 & 0.010 .0611 \\
\hline HIP113084 & -0.080 .076 & -0.170 .054 & -0.050 .035 & 0.000 .086 & -0.070 .053 & 0.080 .044 & 0.090 .0411 \\
\hline HIP113635 & 0.070 .068 & 0.010 .024 & -0.030 .045 & 0.040 .086 & -0.150 .033 & 0.080 .044 & 0.060 .0711 \\
\hline HIP114565 & -0.030 .068 & -0.160 .044 & -0.040 .025 & 0.040 .066 & -0.100 .063 & -0.050 .044 & 0.010 .0711 \\
\hline HIP117954 & -0.040 .077 & -0.210 .024 & -0.030 .025 & 0.020 .086 & -0.080 .053 & 0.040 .044 & 0.010 .0711 \\
\hline
\end{tabular}




\begin{tabular}{|c|c|c|c|c|c|c|c|}
\hline Star & {$[\mathrm{Zn} / \mathrm{Fe}] \sigma \mathrm{N}$} & {$[\mathrm{Y} / \mathrm{Fe}] \sigma \mathrm{N}$} & {$[\mathrm{Zr} / \mathrm{Fe}] \sigma \mathrm{N}$} & {$[\mathrm{Ba} / \mathrm{Fe}] \sigma \mathrm{N}$} & {$[\mathrm{La} / \mathrm{Fe}] \sigma \mathrm{N}$} & {$[\mathrm{Nd} / \mathrm{Fe}] \sigma \mathrm{N}$} & {$[\mathrm{Eu} / \mathrm{Fe}] \sigma \mathrm{N}$} \\
\hline HIP1421 & -0.070 .092 & 0.130 .065 & 0.050 .062 & 0.080 .012 & 0.170 .064 & 0.250 .033 & 0.150 .001 \\
\hline HIP2320 & -0.050 .112 & 0.110 .075 & -0.090 .132 & -0.020 .042 & 0.070 .124 & 0.110 .033 & 0.120 .001 \\
\hline HIP6939 & 0.180 .272 & 0.130 .013 & -0.350 .182 & -0.190 .012 & -0.120 .053 & 0.000 .083 & 0.070 .001 \\
\hline HIP13887 & -0.070 .052 & 0.170 .035 & 0.030 .052 & 0.190 .052 & 0.170 .104 & 0.250 .083 & 0.120 .001 \\
\hline HIP17752 & -0.070 .062 & 0.120 .085 & 0.030 .012 & 0.250 .042 & 0.300 .084 & 0.460 .093 & 0.150 .001 \\
\hline HIP18565 & 0.000 .122 & 0.230 .075 & -0.020 .072 & 0.120 .052 & 0.180 .094 & 0.280 .073 & 0.160 .001 \\
\hline HIP19222 & -0.020 .082 & 0.120 .055 & -0.040 .092 & 0.080 .012 & 0.080 .104 & 0.140 .033 & 0.090 .001 \\
\hline HIP19641 & -0.040 .122 & 0.090 .075 & -0.080 .052 & 0.110 .022 & 0.120 .084 & 0.240 .063 & 0.140 .001 \\
\hline HIP24633 & 0.080 .012 & 0.110 .045 & -0.070 .102 & 0.070 .032 & 0.040 .104 & 0.120 .043 & 0.100 .001 \\
\hline HIP35317 & -0.030 .042 & 0.170 .044 & 0.010 .062 & 0.180 .032 & 0.180 .084 & 0.290 .063 & 0.190 .001 \\
\hline HIP34043 & 0.050 .022 & 0.180 .055 & -0.090 .112 & 0.070 .012 & 0.080 .104 & 0.130 .063 & 0.100 .001 \\
\hline HIP36739 & -0.010 .062 & 0.240 .105 & -0.030 .062 & 0.240 .012 & 0.220 .084 & 0.330 .073 & 0.140 .001 \\
\hline HIP41172 & 0.150 .142 & 0.100 .024 & -0.170 .152 & -0.040 .012 & 0.020 .084 & 0.050 .013 & 0.170 .001 \\
\hline HIP49163 & 0.040 .052 & 0.150 .074 & -0.060 .052 & 0.160 .042 & 0.130 .084 & 0.260 .012 & 0.130 .001 \\
\hline HIP51091 & 0.080 .142 & 0.160 .054 & -0.150 .112 & 0.000 .012 & 0.100 .074 & 0.130 .022 & 0.150 .001 \\
\hline HIP51224 & 0.080 .032 & 0.010 .064 & -0.130 .072 & 0.110 .012 & 0.020 .084 & 0.210 .012 & - - - \\
\hline HIP56756 & 0.080 .082 & 0.170 .094 & -0.160 .152 & -0.010 .012 & 0.050 .084 & 0.120 .012 & 0.150 .001 \\
\hline HIP62405 & -0.010 .022 & 0.130 .104 & 0.000 .152 & 0.120 .032 & 0.050 .104 & 0.000 .022 & 0.100 .001 \\
\hline HIP65366 & 0.080 .062 & 0.120 .044 & -0.030 .062 & 0.140 .032 & 0.150 .094 & 0.240 .022 & 0.210 .001 \\
\hline HIP74080 & 0.000 .102 & 0.160 .055 & 0.060 .072 & 0.170 .012 & 0.210 .074 & 0.270 .032 & 0.130 .001 \\
\hline HIP79164 & 0.040 .102 & 0.170 .064 & -0.050 .072 & 0.100 .012 & 0.140 .084 & 0.210 .012 & 0.190 .001 \\
\hline HIP79647 & 0.100 .162 & 0.180 .074 & -0.100 .092 & 0.080 .012 & 0.110 .094 & 0.180 .063 & 0.070 .001 \\
\hline HIP79867 & 0.160 .222 & 0.460 .053 & -0.310 .232 & -0.040 .052 & 0.030 .084 & 0.110 .012 & 0.090 .001 \\
\hline HIP80656 & -0.010 .072 & 0.190 .064 & -0.040 .102 & 0.160 .042 & 0.160 .084 & 0.290 .052 & 0.090 .001 \\
\hline HIP80839 & 0.010 .052 & 0.100 .054 & -0.060 .142 & 0.060 .032 & 0.070 .084 & 0.100 .032 & 0.090 .001 \\
\hline HIP82219 & 0.010 .042 & 0.170 .044 & 0.030 .072 & 0.190 .022 & 0.180 .064 & 0.240 .032 & 0.160 .001 \\
\hline HIP83289 & 0.080 .102 & 0.100 .044 & -0.110 .152 & 0.010 .012 & 0.090 .114 & 0.120 .012 & 0.170 .001 \\
\hline HIP85160 & 0.000 .062 & 0.170 .054 & 0.020 .092 & 0.210 .012 & 0.190 .074 & 0.250 .043 & 0.140 .001 \\
\hline HIP88204 & 0.160 .172 & 0.080 .064 & -0.270 .142 & -0.080 .012 & 0.080 .124 & 0.080 .032 & 0.140 .001 \\
\hline HIP93589 & 0.140 .042 & 0.040 .064 & -0.190 .072 & 0.060 .012 & 0.080 .064 & 0.140 .012 & 0.090 .001 \\
\hline HIP113084 & 0.170 .152 & 0.070 .075 & -0.270 .152 & -0.110 .012 & 0.020 .114 & 0.090 .083 & 0.120 .001 \\
\hline HIP113635 & 0.120 .052 & -0.050 .115 & -0.300 .102 & -0.010 .042 & -0.020 .064 & 0.210 .093 & 0.270 .001 \\
\hline HIP114565 & -0.130 .052 & 0.130 .045 & -0.000 .142 & 0.100 .012 & 0.120 .084 & 0.140 .013 & 0.070 .001 \\
\hline HIP117954 & 0.040 .222 & 0.180 .095 & -0.180 .152 & -0.020 .012 & 0.070 .104 & 0.160 .083 & 0.130 .001 \\
\hline
\end{tabular}


Table 7: Derived abundances of giants from the Sirius supercluster.

\begin{tabular}{|c|c|c|c|c|c|c|c|c|}
\hline Star & {$[\mathrm{FeI} / \mathrm{H}] \sigma \mathrm{N}$} & {$[\mathrm{FeII} / \mathrm{H}] \sigma \mathrm{N}$} & {$[\mathrm{Na} / \mathrm{Fe}] \sigma \mathrm{N}$} & {$[\mathrm{Mg} / \mathrm{Fe}] \sigma \mathrm{N}$} & {$[\mathrm{Al} / \mathrm{Fe}] \sigma \mathrm{N}$} & {$[\mathrm{Si} / \mathrm{Fe}] \sigma \mathrm{N}$} & {$[\mathrm{Ca} / \mathrm{Fe}] \sigma \mathrm{N}$} & {$[\mathrm{Sc} / \mathrm{Fe}] \sigma \mathrm{N}$} \\
\hline HIP7668 & $\begin{array}{llll}-0.09 & 0.10 & 75\end{array}$ & $\begin{array}{llll}-0.09 & 0.08 & 11\end{array}$ & $\begin{array}{llll}0.19 & 0.02 & 2\end{array}$ & $\begin{array}{llll}-0.03 & 0.07 & 4\end{array}$ & $\begin{array}{llll}0.02 & 0.02 & 4\end{array}$ & $\begin{array}{llll}0.18 & 0.07 & 4\end{array}$ & -0.170 .058 & -0.090 .015 \\
\hline HIP26711 & 0.140 .0787 & 0.130 .0612 & 0.210 .012 & -0.080 .044 & 0.000 .024 & 0.040 .055 & 0.030 .058 & -0.070 .036 \\
\hline HIP28556 & 0.060 .0886 & 0.060 .0711 & 0.100 .032 & -0.030 .044 & 0.040 .034 & 0.090 .065 & -0.060 .058 & -0.040 .046 \\
\hline HIP35431 & 0.000 .0786 & 0.000 .0612 & 0.170 .032 & -0.070 .032 & -0.030 .024 & -0.010 .065 & 0.050 .068 & -0.080 .034 \\
\hline HIP37030 & -0.100 .0886 & -0.110 .0911 & 0.150 .012 & -0.020 .034 & 0.050 .014 & 0.110 .055 & -0.040 .048 & -0.020 .035 \\
\hline HIP47719 & 0.100 .0883 & 0.090 .0811 & 0.110 .012 & -0.020 .044 & 0.040 .024 & 0.050 .065 & -0.090 .068 & -0.070 .046 \\
\hline HIP49103 & 0.030 .0983 & $0.020 .07 \quad 11$ & 0.200 .012 & 0.080 .024 & 0.140 .044 & 0.130 .085 & $\begin{array}{ll}-0.09 & 0.068\end{array}$ & -0.030 .056 \\
\hline HIP51825 & 0.150 .0785 & 0.140 .0611 & 0.250 .022 & -0.030 .044 & -0.010 .014 & 0.010 .075 & 0.060 .058 & -0.040 .026 \\
\hline HIP52926 & $\begin{array}{lll}0.06 & 0.0688\end{array}$ & 0.040 .0611 & 0.090 .022 & -0.040 .054 & 0.020 .044 & 0.030 .065 & 0.040 .068 & -0.050 .026 \\
\hline HIP53710 & $\begin{array}{lll}0.06 & 0.1082\end{array}$ & 0.060 .0910 & 0.130 .012 & 0.000 .054 & 0.120 .044 & 0.100 .065 & -0.090 .058 & -0.020 .056 \\
\hline HIP53876 & $\begin{array}{lll}0.10 & 0.0686\end{array}$ & 0.100 .0510 & 0.210 .022 & -0.020 .034 & -0.020 .034 & -0.010 .075 & 0.040 .058 & -0.100 .036 \\
\hline HIP66567 & $0.00 \quad 0.0786$ & $\begin{array}{ll}0.00 & 0.0511\end{array}$ & 0.030 .052 & 0.040 .044 & 0.050 .044 & 0.060 .065 & 0.000 .068 & -0.020 .026 \\
\hline HIP67021 & $\begin{array}{lll}-0.01 & 0.1079\end{array}$ & $\begin{array}{lll}-0.02 & 0.09 & 10\end{array}$ & 0.300 .022 & 0.070 .044 & 0.210 .044 & 0.160 .045 & -0.050 .068 & -0.050 .046 \\
\hline HIP67364 & 0.080 .0986 & 0.070 .0612 & 0.190 .102 & 0.040 .064 & 0.090 .034 & 0.110 .085 & -0.040 .078 & -0.040 .036 \\
\hline HIP68590 & $\begin{array}{lll}-0.03 & 0.0788\end{array}$ & $\begin{array}{lll}-0.05 & 0.0511\end{array}$ & $\begin{array}{ll}0.10 & 0.022\end{array}$ & 0.040 .034 & 0.070 .034 & 0.090 .055 & 0.030 .068 & 0.010 .036 \\
\hline HIP68737 & 0.080 .0886 & 0.070 .0611 & 0.190 .022 & -0.010 .044 & $0.08 \quad 0.034$ & 0.090 .065 & 0.010 .068 & 0.010 .046 \\
\hline HIP68828 & $0.08 \quad 0.0688$ & 0.070 .0511 & 0.150 .022 & 0.010 .034 & 0.020 .024 & 0.050 .075 & 0.060 .068 & -0.050 .036 \\
\hline HIP68935 & $\begin{array}{lll}-0.10 & 0.07 & 89\end{array}$ & $\begin{array}{lll}-0.11 & 0.0512\end{array}$ & 0.100 .032 & 0.080 .024 & 0.150 .024 & 0.070 .075 & 0.060 .058 & -0.020 .036 \\
\hline HIP77401 & 0.030 .1081 & $\begin{array}{lll}0.03 & 0.1011\end{array}$ & 0.030 .012 & 0.110 .034 & 0.140 .024 & 0.160 .045 & -0.170 .058 & -0.100 .056 \\
\hline HIP79326 & -0.210 .0885 & -0.220 .0911 & $\begin{array}{ll}0.12 & 0.022\end{array}$ & 0.150 .034 & 0.230 .034 & 0.150 .034 & 0.020 .048 & 0.000 .046 \\
\hline HIP80211 & -0.070 .0687 & $\begin{array}{lll}-0.08 & 0.06 & 11\end{array}$ & $\begin{array}{lll}0.05 & 0.032\end{array}$ & $\begin{array}{lll}0.01 & 0.014\end{array}$ & 0.080 .034 & 0.080 .075 & 0.060 .058 & 0.030 .036 \\
\hline HIP117938 & 0.070 .0983 & 0.070 .0711 & 0.210 .012 & 0.060 .054 & 0.120 .034 & 0.110 .065 & -0.090 .057 & -0.040 .036 \\
\hline
\end{tabular}




\begin{tabular}{|c|c|c|c|c|c|c|c|}
\hline Star & {$[\mathrm{TiI} / \mathrm{Fe}] \sigma \mathrm{N}$} & {$[\mathrm{TiII} / \mathrm{Fe}] \sigma \mathrm{N}$} & {$[\mathrm{V} / \mathrm{Fe}] \sigma \mathrm{N}$} & {$[\mathrm{Cr} / \mathrm{Fe}] \sigma \mathrm{N}$} & {$[\mathrm{Mn} / \mathrm{Fe}] \sigma \mathrm{N}$} & {$[\mathrm{Co} / \mathrm{Fe}] \sigma \mathrm{N}$} & {$[\mathrm{Ni} / \mathrm{Fe}] \sigma \mathrm{N}$} \\
\hline HIP7668 & -0.190 .068 & -0.100 .024 & -0.220 .075 & -0.060 .095 & -0.290 .073 & -0.010 .044 & -0.040 .0611 \\
\hline HIP26711 & -0.030 .056 & -0.070 .034 & -0.050 .025 & -0.020 .066 & -0.160 .043 & -0.080 .044 & $\begin{array}{lll}-0.07 & 0.0511\end{array}$ \\
\hline HIP28556 & -0.080 .065 & -0.090 .044 & -0.110 .045 & -0.020 .086 & -0.210 .073 & -0.010 .054 & $\begin{array}{lll}-0.06 & 0.05 & 11\end{array}$ \\
\hline HIP35431 & -0.050 .046 & -0.010 .064 & -0.040 .045 & -0.010 .066 & -0.220 .103 & -0.070 .054 & -0.110 .0611 \\
\hline HIP37030 & -0.050 .067 & -0.070 .054 & -0.110 .045 & -0.040 .066 & -0.230 .053 & -0.040 .054 & -0.060 .0411 \\
\hline HIP47719 & -0.090 .088 & -0.150 .054 & -0.090 .045 & -0.010 .085 & -0.190 .073 & -0.050 .034 & $\begin{array}{lll}-0.02 & 0.06 & 11\end{array}$ \\
\hline HIP49103 & -0.080 .047 & -0.160 .064 & -0.090 .045 & -0.030 .065 & -0.190 .033 & 0.050 .054 & 0.010 .0711 \\
\hline HIP51825 & 0.010 .058 & -0.040 .044 & -0.030 .035 & -0.010 .045 & -0.120 .023 & -0.070 .034 & $\begin{array}{lll}-0.06 & 0.05 & 11\end{array}$ \\
\hline HIP52926 & -0.010 .058 & -0.030 .044 & -0.030 .035 & 0.010 .076 & -0.120 .063 & -0.070 .034 & -0.040 .0611 \\
\hline HIP 53710 & -0.050 .047 & -0.170 .044 & -0.070 .025 & 0.000 .085 & -0.240 .083 & 0.010 .054 & $\begin{array}{lll}-0.01 & 0.08 & 10\end{array}$ \\
\hline HIP 53876 & -0.000 .057 & -0.050 .044 & -0.000 .035 & 0.010 .056 & -0.100 .043 & -0.070 .044 & -0.080 .0611 \\
\hline HIP66567 & -0.040 .068 & -0.030 .044 & -0.040 .025 & 0.000 .076 & -0.110 .073 & -0.030 .034 & $\begin{array}{lll}-0.02 & 0.0511\end{array}$ \\
\hline HIP67021 & -0.020 .067 & -0.180 .014 & -0.020 .055 & 0.080 .056 & -0.170 .073 & 0.050 .064 & 0.010 .079 \\
\hline HIP67364 & -0.080 .066 & -0.160 .024 & -0.100 .045 & 0.030 .086 & -0.140 .053 & 0.000 .034 & 0.010 .0611 \\
\hline HIP68590 & 0.010 .056 & -0.030 .054 & -0.010 .035 & 0.010 .076 & -0.130 .043 & 0.010 .034 & 0.000 .0611 \\
\hline HIP68737 & -0.060 .056 & -0.070 .044 & -0.060 .055 & 0.010 .076 & -0.110 .063 & -0.010 .044 & 0.030 .0411 \\
\hline HIP68828 & -0.030 .057 & -0.030 .054 & -0.050 .045 & 0.010 .076 & -0.160 .023 & -0.070 .034 & -0.050 .0611 \\
\hline HIP68935 & 0.040 .057 & 0.010 .054 & 0.020 .044 & 0.020 .046 & -0.120 .063 & 0.010 .044 & 0.010 .0611 \\
\hline HIP77401 & -0.100 .036 & -0.100 .024 & -0.120 .065 & -0.010 .046 & -0.240 .073 & -0.010 .064 & -0.020 .079 \\
\hline HIP79326 & 0.050 .067 & -0.040 .054 & 0.020 .045 & 0.040 .046 & -0.170 .043 & 0.040 .064 & -0.010 .059 \\
\hline HIP80211 & 0.010 .056 & 0.020 .044 & 0.000 .035 & -0.010 .056 & -0.150 .033 & 0.070 .044 & -0.050 .0611 \\
\hline HIP117938 & -0.090 .046 & -0.160 .054 & -0.100 .035 & 0.020 .066 & -0.220 .073 & 0.010 .034 & 0.000 .0611 \\
\hline
\end{tabular}


Table 9: Derived abundances of giants from the Sirius supercluster.

\begin{tabular}{|c|c|c|c|c|c|c|c|}
\hline Star & {$[\mathrm{Zn} / \mathrm{Fe}] \sigma \mathrm{N}$} & {$[\mathrm{Y} / \mathrm{Fe}] \sigma \mathrm{N}$} & {$[\mathrm{Zr} / \mathrm{Fe}] \sigma \mathrm{N}$} & {$[\mathrm{Ba} / \mathrm{Fe}] \sigma \mathrm{N}$} & {$[\mathrm{La} / \mathrm{Fe}] \sigma \mathrm{N}$} & {$[\mathrm{Nd} / \mathrm{Fe}] \sigma \mathrm{N}$} & {$[\mathrm{Eu} / \mathrm{Fe}] \sigma \mathrm{N}$} \\
\hline HIP7668 & 0.020 .302 & 0.460 .104 & -0.270 .132 & 0.260 .052 & 0.220 .224 & 0.230 .032 & 0.090 .001 \\
\hline HIP26711 & -0.060 .072 & 0.140 .095 & 0.140 .022 & 0.300 .032 & 0.180 .084 & 0.310 .053 & 0.100 .001 \\
\hline HIP28556 & 0.060 .102 & 0.340 .185 & -0.050 .012 & 0.170 .022 & 0.210 .144 & 0.440 .193 & 0.120 .001 \\
\hline HIP35431 & -0.080 .072 & 0.150 .065 & 0.260 .012 & 0.380 .042 & 0.210 .104 & 0.370 .063 & 0.130 .001 \\
\hline HIP37030 & -0.020 .192 & 0.290 .154 & -0.040 .032 & 0.290 .042 & 0.310 .134 & 0.480 .123 & 0.170 .001 \\
\hline HIP47719 & $\begin{array}{lll}-0.01 & 0.21 & 2\end{array}$ & 0.260 .145 & -0.140 .112 & 0.110 .012 & 0.180 .134 & 0.370 .153 & 0.130 .001 \\
\hline HIP49103 & 0.090 .212 & 0.400 .084 & -0.260 .152 & 0.040 .012 & 0.060 .124 & 0.150 .052 & 0.160 .001 \\
\hline HIP51825 & -0.070 .042 & 0.150 .065 & 0.070 .072 & 0.250 .062 & 0.210 .084 & 0.240 .023 & 0.130 .001 \\
\hline HIP52926 & -0.080 .032 & 0.160 .075 & 0.060 .042 & 0.260 .052 & 0.230 .094 & 0.380 .053 & 0.130 .001 \\
\hline HIP53710 & 0.000 .202 & 0.610 .124 & -0.120 .152 & 0.210 .042 & 0.240 .184 & 0.280 .052 & 0.110 .001 \\
\hline HIP53876 & -0.100 .012 & 0.140 .095 & 0.120 .042 & 0.280 .032 & 0.170 .094 & 0.210 .043 & 0.110 .001 \\
\hline HIP66567 & 0.000 .102 & 0.140 .065 & -0.070 .072 & 0.100 .062 & 0.160 .074 & 0.320 .093 & 0.120 .001 \\
\hline HIP67021 & 0.160 .222 & 0.540 .114 & -0.160 .182 & 0.060 .012 & 0.080 .154 & 0.160 .072 & 0.080 .001 \\
\hline HIP 67364 & $\begin{array}{lll}0.10 & 0.202\end{array}$ & 0.230 .135 & -0.180 .132 & 0.040 .012 & 0.080 .174 & 0.250 .143 & 0.140 .001 \\
\hline HIP68590 & 0.010 .112 & 0.120 .055 & -0.010 .012 & 0.170 .042 & 0.160 .084 & 0.300 .023 & 0.220 .001 \\
\hline HIP68737 & 0.090 .132 & 0.090 .045 & -0.110 .092 & 0.060 .012 & 0.050 .114 & 0.180 .073 & 0.110 .001 \\
\hline HIP68828 & -0.040 .052 & 0.160 .065 & 0.120 .032 & 0.280 .042 & 0.240 .054 & 0.380 .043 & 0.150 .001 \\
\hline HIP68935 & 0.050 .042 & 0.120 .035 & -0.050 .042 & 0.100 .012 & 0.130 .074 & 0.290 .083 & 0.100 .001 \\
\hline HIP77401 & 0.070 .212 & 0.340 .114 & -0.340 .122 & 0.020 .032 & -0.010 .164 & 0.100 .112 & 0.170 .001 \\
\hline HIP79326 & 0.180 .142 & 0.360 .084 & -0.210 .082 & -0.010 .012 & 0.050 .094 & 0.230 .112 & 0.170 .001 \\
\hline HIP80211 & -0.040 .022 & 0.160 .075 & 0.070 .012 & 0.300 .072 & 0.250 .094 & 0.420 .083 & 0.170 .001 \\
\hline HIP117938 & 0.050 .192 & 0.420 .104 & -0.190 .122 & 0.070 .032 & 0.070 .154 & 0.160 .052 & 0.110 .001 \\
\hline
\end{tabular}




\begin{tabular}{|c|c|c|c|c|c|c|c|c|}
\hline \multirow[b]{2}{*}{ Entity } & \multicolumn{4}{|c|}{ Hyades (Melotte 25) } & \multicolumn{4}{|c|}{ Praesepe (NGC 2632) } \\
\hline & $\gamma \mathrm{Tau}$ & $\delta \mathrm{Tau}$ & $\varepsilon \mathrm{Tau}$ & Mean & HD 73598 & HD 73665 & HD 73710 & Mean \\
\hline 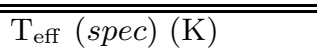 & 4960 & 4950 & 4890 & & 5000 & 5020 & 4920 & \\
\hline $\log g($ spec $)\left(\mathrm{cm} \mathrm{s}^{-1}\right)$ & 2.68 & 2.60 & 2.55 & & 2.70 & 2.68 & 2.58 & \\
\hline$\xi_{t}\left(\mathrm{~km} \mathrm{~s}^{-1}\right.$ & 1.61 & 1.59 & 1.70 & & 1.58 & 1.70 & 1.70 & \\
\hline$[\mathrm{M} / \mathrm{H}]_{\text {model }}$ & +0.15 & +0.15 & +0.13 & & +0.17 & +0.20 & +0.18 & \\
\hline$[\mathrm{NaI} / \mathrm{Fe}]$ & 0.330 .012 & 0.350 .012 & 0.380 .032 & 0.350 .03 & 0.340 .012 & 0.410 .012 & 0.390 .022 & 0.380 .04 \\
\hline$[\mathrm{MgI} / \mathrm{Fe}]$ & -0.030 .043 & -0.060 .043 & -0.040 .034 & -0.040 .02 & -0.070 .054 & -0.060 .064 & -0.090 .043 & -0.070 .02 \\
\hline$[\mathrm{AlI} / \mathrm{Fe}]$ & 0.080 .064 & 0.070 .054 & 0.110 .054 & 0.090 .02 & 0.070 .044 & 0.070 .054 & 0.090 .064 & 0.080 .01 \\
\hline$[\mathrm{SiI} / \mathrm{Fe}]$ & 0.040 .095 & 0.040 .095 & 0.090 .075 & $0.06 \quad 0.03$ & 0.060 .075 & 0.070 .085 & 0.070 .085 & 0.070 .01 \\
\hline$[\mathrm{CaI} / \mathrm{Fe}]$ & 0.010 .058 & 0.020 .078 & 0.020 .058 & 0.020 .01 & 0.020 .078 & 0.010 .068 & -0.010 .078 & 0.010 .02 \\
\hline$[\mathrm{ScII} / \mathrm{Fe}]$ & -0.080 .066 & -0.100 .066 & -0.060 .056 & -0.080 .02 & -0.070 .046 & -0.050 .056 & -0.070 .066 & -0.060 .01 \\
\hline$[\mathrm{TiI} / \mathrm{Fe}]$ & -0.060 .068 & -0.080 .057 & -0.030 .058 & -0.060 .03 & -0.030 .066 & -0.060 .057 & -0.070 .067 & -0.050 .02 \\
\hline$[\mathrm{TiII} / \mathrm{Fe}]$ & -0.140 .044 & -0.110 .044 & -0.120 .034 & -0.120 .02 & -0.100 .044 & -0.090 .064 & -0.120 .074 & -0.100 .02 \\
\hline$[\mathrm{VI} / \mathrm{Fe}]$ & -0.040 .045 & -0.080 .045 & -0.030 .065 & -0.050 .03 & 0.000 .045 & -0.030 .055 & -0.030 .045 & $-0.02 \quad 0.02$ \\
\hline$[\mathrm{CrI} / \mathrm{Fe}]$ & -0.010 .095 & -0.020 .085 & 0.030 .105 & 0.000 .03 & 0.010 .076 & 0.020 .086 & -0.010 .086 & 0.000 .02 \\
\hline$[\mathrm{MnI} / \mathrm{Fe}]$ & -0.120 .013 & -0.130 .013 & -0.060 .083 & -0.100 .04 & -0.070 .043 & -0.100 .043 & -0.110 .063 & -0.090 .02 \\
\hline$[\mathrm{FeI} / \mathrm{H}]$ & 0.160 .0886 & 0.160 .0883 & 0.130 .0986 & 0.150 .02 & 0.180 .0784 & 0.210 .0783 & 0.180 .0884 & 0.190 .02 \\
\hline$[\mathrm{FeII} / \mathrm{H}]$ & 0.140 .0612 & 0.140 .0612 & 0.110 .0510 & 0.130 .02 & 0.160 .0711 & $0.190 .07 \quad 12$ & 0.170 .0411 & 0.170 .02 \\
\hline$[\mathrm{CoI} / \mathrm{Fe}]$ & -0.050 .054 & -0.080 .064 & -0.020 .044 & -0.050 .03 & -0.040 .044 & -0.040 .044 & -0.050 .064 & -0.040 .01 \\
\hline$[\mathrm{NiI} / \mathrm{Fe}]$ & $0.00 \quad 0.0612$ & 0.000 .0711 & 0.030 .0611 & 0.010 .02 & 0.010 .0612 & 0.020 .0712 & 0.040 .0612 & 0.020 .02 \\
\hline$[\mathrm{ZnI} / \mathrm{Fe}]$ & -0.030 .152 & -0.060 .122 & 0.010 .082 & -0.030 .04 & -0.030 .102 & -0.030 .132 & 0.010 .062 & -0.020 .02 \\
\hline$[\mathrm{YII} / \mathrm{Fe}]$ & 0.040 .084 & -0.020 .045 & 0.050 .095 & 0.020 .04 & 0.020 .065 & 0.030 .065 & 0.040 .055 & 0.030 .01 \\
\hline$[\mathrm{ZrI} / \mathrm{Fe}]$ & -0.040 .142 & -0.080 .172 & -0.050 .162 & -0.060 .02 & -0.030 .142 & -0.050 .142 & -0.050 .162 & -0.040 .01 \\
\hline$[\mathrm{BaII} / \mathrm{Fe}]$ & 0.060 .022 & 0.030 .012 & 0.010 .012 & 0.030 .03 & 0.050 .012 & 0.030 .012 & 0.040 .012 & 0.040 .01 \\
\hline$[\mathrm{LaII} / \mathrm{Fe}]$ & 0.010 .144 & -0.020 .114 & $0.01 \quad 0.124$ & 0.000 .02 & 0.040 .104 & $0.03 \quad 0.124$ & 0.070 .124 & 0.050 .02 \\
\hline$[\mathrm{NdII} / \mathrm{Fe}]$ & 0.010 .063 & 0.000 .033 & 0.060 .053 & 0.020 .03 & 0.060 .023 & 0.070 .013 & 0.050 .033 & 0.060 .01 \\
\hline$[\mathrm{EuII} / \mathrm{Fe}]$ & $0.04-1$ & $0.05-1$ & $0.06-1$ & 0.050 .01 & $0.08-1$ & $0.08-1$ & $0.08-1$ & 0.080 .01 \\
\hline
\end{tabular}


Table 11: Mean abundances for the sample of open clusters, the Hyades and Sirius supercluster giants. The samples are divided into two or three $[\mathrm{Fe} / \mathrm{H}]$ bins as indicated in the table and $\mathrm{N}$ is the number of stars in a bin.

\begin{tabular}{|c|c|c|c|c|c|c|c|}
\hline \multirow{2}{*}{$\begin{array}{l}\text { Entry } \\
\mathrm{N}\end{array}$} & \multicolumn{2}{|c|}{ Open clusters } & \multicolumn{3}{|c|}{ Hyades supercluster } & \multicolumn{2}{|c|}{ Sirius supercluster } \\
\hline & 16 & 18 & 7 & 12 & 15 & 8 & 14 \\
\hline$[\mathrm{Fe} / \mathrm{H}]$ & -0.180 .09 & -0.050 .05 & -0.090 .07 & 0.040 .02 & 0.170 .04 & -0.070 .07 & 0.070 .04 \\
\hline$[\mathrm{Na} / \mathrm{Fe}]$ & 0.240 .09 & 0.240 .09 & 0.110 .03 & 0.170 .05 & 0.270 .06 & 0.130 .09 & 0.160 .06 \\
\hline$[\mathrm{Mg} / \mathrm{Fe}]$ & 0.050 .13 & 0.020 .07 & 0.050 .08 & 0.000 .04 & 0.000 .03 & 0.040 .06 & 0.000 .06 \\
\hline$[\mathrm{Al} / \mathrm{Fe}]$ & 0.040 .10 & 0.060 .04 & 0.140 .05 & 0.080 .03 & 0.100 .05 & 0.110 .08 & 0.050 .06 \\
\hline$[\mathrm{Si} / \mathrm{Fe}]$ & 0.180 .08 & 0.160 .07 & 0.120 .05 & 0.080 .02 & 0.090 .03 & 0.110 .05 & 0.060 .05 \\
\hline$[\mathrm{Ca} / \mathrm{Fe}]$ & 0.020 .09 & 0.030 .06 & 0.040 .03 & 0.010 .03 & -0.020 .04 & -0.010 .08 & -0.020 .07 \\
\hline$[\mathrm{Sc} / \mathrm{Fe}]$ & 0.040 .08 & 0.020 .06 & -0.010 .04 & -0.040 .03 & -0.030 .03 & -0.020 .04 & -0.050 .03 \\
\hline$[\mathrm{Ti} / \mathrm{Fe}]$ & -0.020 .12 & -0.020 .05 & 0.000 .04 & -0.040 .03 & -0.070 .03 & -0.040 .06 & -0.070 .04 \\
\hline$[\mathrm{V} / \mathrm{Fe}]$ & 0.000 .09 & 0.030 .06 & -0.030 .03 & -0.040 .03 & -0.040 .02 & -0.050 .08 & -0.070 .04 \\
\hline$[\mathrm{Cr} / \mathrm{Fe}]$ & 0.010 .04 & 0.030 .04 & 0.000 .03 & 0.010 .01 & 0.010 .02 & 0.000 .04 & 0.000 .02 \\
\hline$[\mathrm{Mn} / \mathrm{Fe}]$ & -0.090 .09 & -0.080 .05 & -0.110 .04 & -0.080 .03 & -0.090 .03 & -0.170 .06 & -0.170 .05 \\
\hline$[\mathrm{Co} / \mathrm{Fe}]$ & 0.030 .09 & 0.050 .06 & 0.010 .05 & -0.020 .03 & 0.020 .06 & -0.070 .07 & 0.070 .04 \\
\hline$[\mathrm{Ni} / \mathrm{Fe}]$ & -0.020 .05 & 0.010 .04 & 0.010 .03 & -0.010 .02 & 0.050 .03 & 0.010 .04 & -0.030 .04 \\
\hline$[\mathrm{Zn} / \mathrm{Fe}]$ & -0.150 .11 & -0.090 .09 & 0.050 .07 & 0.000 .04 & 0.060 .10 & 0.050 .08 & 0.000 .07 \\
\hline$[\mathrm{Y} / \mathrm{Fe}]$ & 0.080 .05 & 0.070 .06 & 0.090 .10 & 0.160 .04 & 0.120 .03 & 0.170 .07 & 0.160 .05 \\
\hline$[\mathrm{Zr} / \mathrm{Fe}]$ & 0.070 .10 & 0.080 .08 & $\begin{array}{lll}-0.10 & 0.11\end{array}$ & -0.030 .07 & -0.120 .11 & -0.040 .08 & 0.000 .15 \\
\hline$[\mathrm{Ba} / \mathrm{Fe}]$ & 0.160 .13 & 0.090 .09 & 0.130 .09 & 0.120 .06 & 0.010 .09 & 0.160 .12 & 0.180 .12 \\
\hline$[\mathrm{La} / \mathrm{Fe}]$ & 0.060 .16 & 0.050 .10 & 0.130 .11 & 0.150 .05 & 0.060 .06 & 0.170 .09 & 0.150 .08 \\
\hline$[\mathrm{Nd} / \mathrm{Fe}]$ & 0.150 .11 & 0.100 .06 & 0.260 .10 & 0.220 .08 & 0.110 .05 & 0.290 .08 & 0.270 .10 \\
\hline$[\mathrm{Eu} / \mathrm{Fe}]$ & 0.160 .09 & 0.080 .06 & 0.170 .06 & 0.130 .04 & 0.120 .03 & 0.140 .05 & 0.130 .03 \\
\hline
\end{tabular}

